\title{
Synchronization and Transient Stability in Power Networks and Non-Uniform Kuramoto Oscillators
}

\author{
Florian Dörfler, Member, IEEE, and Francesco Bullo, Fellow, IEEE
}

\begin{abstract}
Motivated by recent interest for multi-agent systems and smart power grid architectures, we discuss the synchronization problem for the network-reduced model of a power system with non-trivial transfer conductances. Our key insight is to exploit the relationship between the power network model and a first-order model of coupled oscillators. Assuming overdamped generators (possibly due to local excitation controllers), a singular perturbation analysis shows the equivalence between the classic swing equations and a non-uniform Kuramoto model. Here, non-uniform Kuramoto oscillators are characterized by multiple time constants, non-homogeneous coupling, and non-uniform phase shifts. Extending methods from transient stability, synchronization theory, and consensus protocols, we establish sufficient conditions for synchronization of non-uniform Kuramoto oscillators. These conditions reduce to and improve upon previously-available tests for the standard Kuramoto model. Combining our singular perturbation and Kuramoto analyses, we derive concise and purely algebraic conditions that relate synchronization and transient stability of a power network to the underlying system parameters and initial conditions.
\end{abstract}

\section{INTRODUCTION}

The vast North American interconnected power grid is often referred to as the largest and most complex machine engineered by humankind. The various instabilities arising in such a large-scale power grid can be classified by their physical nature, the size of the uncertainty or disturbance causing the instability, or depending on the devices, processes, and the time necessary to determine the instability. All of these instabilities can lead and have led to blackouts of power grids [2], and their detection and rejection will be one of the major challenges faced by the future "smart power grid."

This work was supported in part by NSF grants IIS-0904501 and CNS-0834446. This document is a vastly revised and extended version of [1]. The authors gratefully acknowledge Prof. Yoshihiko Susuki and Prof. Petar Kokotović for their insightful comments that improved the presentation of this article.

Florian Dörfler and Francesco Bullo are with the Center for Control, Dynamical Systems and Computation, University of California at Santa Barbara, Santa Barbara, CA 93106, \{dorfler, bullo\}@engineering.ucsb. edu 
The envisioned future power generation will rely increasingly on renewable energy sources such as wind and solar power. Since these renewable power sources are highly stochastic, there will be an increasing number of transient disturbances acting on a power grid that is expected to be even more complex and decentralized than the current one. Thus, an important form of power network stability is the so-called transient stability [3], which is the ability of a power system to remain in synchronism when subjected to large transient disturbances. These disturbances may include faults on transmission elements or loss of load, loss of generation, or loss of system components. For example, a recent major blackout in Italy in 2003 was caused by tripping of a tie-line and resulted in a cascade of events leading to the loss of synchronism of the Italian power grid with the rest of Europe [2]. The mechanism by which interconnected synchronous machines maintain synchronism is a balance of their mechanical power inputs and their electrical power outputs depending on the relative rotor angles among machines. In a classic setting the transient stability problem is posed as a special case of the more general synchronization problem, which is defined over a possibly longer time horizon, for rotor angles possibly drifting away from their nominal values, and for generators subject to local excitation controllers aiming to restore synchronism. In order to analyze the stability of a synchronous operating point of a power grid and to estimate its region of attraction, various sophisticated algorithms have been developed [4], [5], [6], [7], [8], [9]. Reviews and survey articles on transient stability analysis can be found in [10], [11], [12], [13]. Unfortunately, the existing methods can cope only with simplified models and do not provide simple formulas to check if a power system synchronizes for a given system state and parameters. In fact, an open problem, recognized by [14] and not resolved by classical analysis methods, is the quest for explicit and concise conditions for synchronization as a function of the topological, algebraic, and spectral graph properties of the network.

The recent years have witnessed a burgeoning interest of the control community in cooperative control of autonomous agent systems. Recent surveys and monographs include [15], [16], [17]. One of the basic tasks in a multi-agent system is a consensus of the agents' states to a common value. This consensus problem has been subject to fundamental research [18], [19] as well as to applications in robotic coordination, distributed sensing and computation, and various other fields including synchronization. In most articles treating consensus problems the agents obey single integrator dynamics, but the synchronization of interconnected power systems has often been envisioned as possible future application [20]. However, we are aware of only one article [21] that indeed applies consensus methods to a power network model.

Another set of literature relevant to our investigation is the synchronization of coupled oscillators [22], in particular in the classic model introduced by Kuramoto [23]. The synchronization of coupled Kuramoto oscillators has been widely studied by the physics [24], [25], [26] and the dynamical systems communities [27], [28], [29]. This vast literature with numerous theoretical results and rich applications to various scientific areas is elegantly reviewed in [30], [31]. Recent works in the control community [18], [19], [32], [33] investigate the close relationship between Kuramoto oscillators and consensus networks.

The three areas of power network synchronization, Kuramoto oscillators, and consensus protocols are 
apparently closely related. Indeed, the similarity between the Kuramoto model and the power network models used in transient stability analysis is striking. Even though power networks have often been referred to as systems of coupled oscillators, the similarity to a second-order Kuramoto-type model has been mentioned only very recently in the power networks community in [34], [35], [36], where only qualitative simulation studies for simplified models are carried out. In the coupled-oscillators literature, second-order Kuramoto models similar to power network models have been analyzed in simulations and in the continuum limit; see [31] and references therein. However, we are aware of only two articles referring to power networks as possible application [22], [37]. In short, the evident relationship between power network synchronization, Kuramoto oscillators, and consensus protocols has been recognized, but the gap between the first and the second two topics has not been bridged yet in a thorough analysis.

There are three main contributions in the present paper. As a first contribution, we present a coupledoscillator approach to the problem of synchronization and transient stability in power networks. Via a singular perturbation analysis, we show that the transient stability analysis for the classic swing equations with overdamped generators reduces, on a long time-scale, to the problem of synchronizing non-uniform Kuramoto oscillators with multiple time constants, non-homogeneous coupling, and non-uniform phaseshifts. This reduction to a non-uniform Kuramoto model is arguably the missing link connecting transient stability analysis to networked control, a link that was hinted at in [14], [20], [34], [35], [36], [22], [21].

Second, we give novel, simple, and purely algebraic conditions that are sufficient for synchronization and transient stability of a power network. To the best of our knowledge these conditions are the first ones to relate synchronization and performance of a power network directly to the underlying network parameters and initial state. Our conditions are based on different and possibly less restrictive assumptions than those obtained by classic analysis methods [4], [5], [6], [7], [8], [9], [10]. We consider a networkreduced model of a power network, and do not make any of the following common or classic assumptions: we do not require the swing equations to be formulated in relative coordinates accompanied by a uniform damping assumption, we do not require the existence of an infinite bus, and we do not require the transfer conductances to be "sufficiently small" or even negligible. On the other hand, our results are based on the assumption that each generator is strongly overdamped, possibly due to internal excitation control. This assumption allows us to perform a singular perturbation analysis and study a dimension-reduced system. Due to topological equivalence, our synchronization conditions hold locally even if generators are not overdamped, and in the application to real power networks the approximation via the dimension-reduced system is theoretically well-studied and also applied in the power industry [38]. Our synchronization conditions are based on an analytic approach whereas classic analysis methods [4], [5], [7], [8], [9], [10] rely on numerical procedures to approximate the region of attraction of an equilibrium by level sets of energy functions and stable manifolds. Compared to classic analysis methods, our analysis does not aim at providing best estimates of the region of attraction or the critical clearing time. Rather, we approach the open problem [14] of relating synchronization to the underlying network structure. For this problem, 
we derive sufficient and purely algebraic conditions that can be interpreted as "the network connectivity has to dominate the network's non-uniformity, the network's losses, and the lack of phase cohesiveness."

Third and final, we perform a synchronization analysis of non-uniform Kuramoto oscillators, as an interesting mathematical problem in its own right. Our analysis combines and extends methods from consensus protocols and synchronization theory. As an outcome, purely algebraic conditions on the network parameters and the system state establish the phase cohesiveness, frequency synchronization, and phase synchronization of the non-uniform Kuramoto oscillators. We emphasize that our results do not hold only for non-uniform network parameters but also in the case when the underlying coupling topology is not a complete graph. When our results are specialized to classic (uniform) Kuramoto oscillators, they reduce to and even improve upon various well-known conditions in the literature on the Kuramoto model [25], [24], [19], [32], [33], [39]. In the end, these conditions guaranteeing synchronization of non-uniform Kuramoto oscillators also suffice for the transient stability of the power network.

Paper Organization: This article is organized as follows. The remainder of this section introduces some notation, recalls preliminaries on algebraic graph theory and differential geometry, and reviews the consensus protocol and the Kuramoto model of coupled oscillators. Section II reviews the problem of transient stability analysis. Section III introduces the non-uniform Kuramoto model and presents the main result of this article. Section IV translates the power network model to the non-uniform Kuramoto model whose synchronization analysis is presented in Section V. Section VI provides simulation studies to illustrate the analytical results. Finally, some conclusions are drawn in Section VII. The appendix in Section VIII contains different synchronization conditions and estimates for the phase cohesiveness that can be derived alternatively to the ones presented in Section V.

Vector and matrix notation: Given an $n$-tuple $\left(x_{1}, \ldots, x_{n}\right), \operatorname{diag}\left(x_{i}\right) \in \mathbb{R}^{n \times n}$ is the associated diagonal matrix, $x \in \mathbb{R}^{n}$ is the associated column vector, $x_{\max }$ and $x_{\min }$ are the maximum and minimum elements, and $\|x\|_{2}$ and $\|x\|_{\infty}$ are the 2- and $\infty$-norm. Let $\mathbf{1}_{n}$ and $\mathbf{0}_{n}$ be the vectors of 1 's and 0 's of dimension $n$. Given two non-zero vectors $x \in \mathbb{R}^{n}$ and $y \in \mathbb{R}^{n}$, the angle $\angle(x, y) \in[0, \pi / 2]$ between them satisfies $\cos (\angle(x, y))=x^{T} y /(\|x\|\|y\|)$. Given an array $\left\{A_{i j}\right\}$ with $i, j \in\{1, \ldots, n\}, A \in \mathbb{R}^{n \times n}$ is the associated matrix with $A_{\max }=\max _{i, j}\left\{A_{i j}\right\}$ and $A_{\min }=\min _{i, j}\left\{A_{i j}\right\}$. Given a total order relation among the indices $(i, j)$, let $\operatorname{diag}\left(A_{i j}\right)$ denote the corresponding diagonal matrix.

Graph theory: A weighted directed graph is a triple $\mathcal{G}=(\mathcal{V}, \mathcal{E}, A)$, where $\mathcal{V}=\{1, \ldots, n\}$ is the set of nodes, $\mathcal{E} \subset \mathcal{V} \times \mathcal{V}$ is the set of directed edges, and $A \in \mathbb{R}^{n \times n}$ is the adjacency matrix. The entries of $A$ satisfy $a_{i j}>0$ for each directed edge $(i, j) \in \mathcal{E}$ and are zero otherwise. Any nonnegative matrix $A$ induces a weighted directed graph $\mathcal{G}$. The Laplacian of $\mathcal{G}$ is the $n \times n$ matrix $L\left(a_{i j}\right):=\operatorname{diag}\left(\sum_{j=1}^{n} a_{i j}\right)-A$. In the following, we assume that $A=A^{T}$, that is, $\mathcal{G}$ is undirected. In this case, the graph $\mathcal{G}$ is fully described by the elements $a_{i j}$ with $i \geq j$. If a number $k \in\{1, \ldots,|\mathcal{E}|\}$ and a weight $w_{k}=a_{i j}$ is assigned to any of these edges $(i, j)$ with $i>j$, then the incidence matrix $H \in \mathbb{R}^{|\mathcal{E}| \times n}$ is defined component-wise as $H_{k l}=1$ if node $l$ is the sink node of edge $k$ and as $H_{k l}=-1$ if node $l$ is the source node of edge $k$; all 
other elements are zero. The Laplacian equals then the symmetric matrix $L\left(a_{i j}\right)=H^{T} \operatorname{diag}\left(w_{k}\right) H$. If $\mathcal{G}$ is connected, then $\operatorname{ker}(H)=\operatorname{ker}\left(L\left(a_{i j}\right)\right)=\operatorname{span}\left(\mathbf{1}_{n}\right)$, all $n-1$ remaining non-zero eigenvalues of $L(\mathcal{G})$ are strictly positive, and the second-smallest eigenvalue $\lambda_{2}\left(L\left(a_{i j}\right)\right)$ is called the algebraic connectivity of $\mathcal{G}$ and, for a complete and uniformly weighted graph $\left(a_{i j} \equiv 1\right.$ for all $\left.i \neq j\right)$, it satisfies $\lambda_{2}\left(L\left(a_{i j}\right)\right)=n$.

Geometry on the $n$-torus: The torus is the set $\left.\left.\mathbb{T}^{1}=\right]-\pi,+\pi\right]$, where $-\pi$ and $+\pi$ are associated with each other, an angle is an element $\theta \in \mathbb{T}^{1}$, and an $\operatorname{arc}$ is a connected subset of $\mathbb{T}^{1}$. The product set $\mathbb{T}^{n}$ is the $n$-dimensional torus. With slight abuse of notation, let $\left|\theta_{1}-\theta_{2}\right|$ denote the geodesic distance between two angles $\theta_{1} \in \mathbb{T}^{1}$ and $\theta_{2} \in \mathbb{T}^{1}$. For $\gamma \in[0, \pi]$, let $\Delta(\gamma) \subset \mathbb{T}^{n}$ be the set of angle arrays $\left(\theta_{1}, \ldots, \theta_{n}\right) \in \mathbb{T}^{n}$ such that there exists an arc of length $\gamma$ containing all $\theta_{1}, \ldots, \theta_{n}$ in its interior. Thus, an array of angles $\theta \in \Delta(\gamma)$ satisfies $\max _{i, j \in\{1, \ldots, n\}}\left|\theta_{i}-\theta_{j}\right|<\gamma$. For $\gamma \in[0, \pi]$, we also define $\bar{\Delta}(\gamma)$ to be the union of the set $\left\{\theta \in \mathbb{T}^{n} \mid \theta_{i}=\theta_{j}, i, j \in\{1, \ldots, n\}\right\}$ and the closure of the open set $\Delta(\gamma)$.

For a rigorous definition of the difference between angles (i.e., points on the torus), we restrict our attention to angles contained in an open half-circle: for angles $\theta_{1}, \theta_{2}$ with $\left|\theta_{1}-\theta_{2}\right|<\pi$, the difference $\theta_{1}-\theta_{2}$ is the number in ] $-\pi, \pi$ [ with magnitude equal to the geodesic distance $\left|\theta_{1}-\theta_{2}\right|$ and with positive sign iff the counter-clockwise path length connecting $\theta_{1}$ and $\theta_{2}$ on $\mathbb{T}^{1}$ is smaller than the clockwise path length. Finally, we define the multivariable sine $\sin : \mathbb{T}^{n} \rightarrow[0,1]^{n}$ by $\sin (x)=\left(\sin \left(x_{1}\right), \ldots, \sin \left(x_{n}\right)\right)$ and the sinc function $\operatorname{sinc}: \mathbb{R} \rightarrow \mathbb{R}$ by $\operatorname{sinc}(x)=\sin (x) / x$.

Review of the Consensus Protocol and the Kuramoto Model: In a system of $n$ autonomous agents, each characterized by a state variable $x_{i} \in \mathbb{R}$, one of the most basic tasks is to achieve a consensus on a common state value, that is, all agent states $x_{i}(t)$ converge to a common value $x_{\infty} \in \mathbb{R}$ as $t \rightarrow \infty$. Given a graph $\mathcal{G}$ with adjacency matrix $A$ describing the interaction between agents, a simple, linear, and continuous time algorithm to achieve consensus on the agents' state is the consensus protocol

$$
\dot{x}_{i}=-\sum_{j=1}^{n} a_{i j}\left(x_{i}-x_{j}\right), \quad i \in\{1, \ldots, n\} .
$$

In vector notation the consensus protocol (1) takes the form $\dot{x}=-L\left(a_{i j}\right) x$, which directly reveals the dependence of the consensus protocol to the underlying graph $\mathcal{G}$.

A well-known and widely used model for the synchronization among coupled oscillators is the $\mathrm{Ku}$ ramoto model, which considers $n$ coupled oscillators with state $\theta_{i} \in \mathbb{T}^{1}$ with the dynamics

$$
\dot{\theta}_{i}=\omega_{i}-\frac{K}{n} \sum_{j=1}^{n} \sin \left(\theta_{i}-\theta_{j}\right), \quad i \in\{1, \ldots, n\},
$$

where $K$ is the coupling strength and $\omega_{i}$ is the natural frequency of oscillator $i$. Unlike for the consensus protocol (1), different levels of consensus or synchronization can be distinguished for the Kuramoto model (2): The case when all angles $\theta_{i}(t)$ converge to a common angle $\theta_{\infty} \in \mathbb{T}^{1}$ as $t \rightarrow \infty$ is referred to as phase synchronization and can only occur if all natural frequencies are identical. If the natural frequencies are non-identical, then each phase difference $\theta_{i}(t)-\theta_{j}(t)$ can converge to a constant value, but this value is not necessarily zero. A solution $\theta: \mathbb{R}_{\geq 0} \rightarrow \mathbb{T}^{n}$ to the Kuramoto model (2) is phase cohesive if there exists a length $\gamma \in[0, \pi[$ such that $\theta(t) \in \bar{\Delta}(\gamma)$ for all $t \geq 0$, i.e., at each time $t$ there 
exists an arc of length $\gamma$ containing all angles $\theta_{i}(t)$. A solution $\theta: \mathbb{R}_{\geq 0} \rightarrow \mathbb{T}^{n}$ achieves exponential frequency synchronization if all frequencies $\dot{\theta}_{i}(t)$ converge exponentially fast to a common frequency $\dot{\theta}_{\infty} \in \mathbb{R}$ as $t \rightarrow \infty$. Finally, a solution $\theta: \mathbb{R}_{\geq 0} \rightarrow \mathbb{T}^{n}$ achieves exponential synchronization if it is phase cohesive and it achieves exponential frequency synchronization. In this case, all phases become constant in a rotating coordinate frame with frequency $\dot{\theta}_{\infty}$, and hence the terminology phase locking is sometimes also used in the literature.

\section{Models and Problem Setup in Synchronization And Transient Stability Analysis}

\section{A. The Mathematical Model of a Power Network}

In a power network with $n$ generators we associate to each generator its internal voltage $E_{i}>0$, its active power output $P_{\mathrm{e}, i}$, its mechanical power input $P_{\mathrm{m}, i}>0$, its inertia $M_{i}>0$, its damping constant $D_{i}>0$, and its rotor angle $\theta_{i}$ measured with respect to a rotating frame with frequency $f_{0}$. All parameters are given in per unit system, except for $M_{i}$ and $D_{i}$ which are given in seconds, and $f_{0}$ is typically given as $50 \mathrm{~Hz}$ or $60 \mathrm{~Hz}$. The rotor dynamics of generator $i$ are then given by the classic constant-voltage behind reactance model of interconnected swing equations [11], [40], [41]

$$
M_{i} \ddot{\theta}_{i}=P_{\mathrm{m}, i}-E_{i}^{2} G_{i i}-D_{i} \dot{\theta}_{i}-P_{\mathrm{e}, i}, \quad i \in\{1, \ldots, n\} .
$$

Under the common assumption that the loads are modeled as passive admittances, all passive nodes of a power network can be eliminated (c.f. Kron reduction [42]) resulting in the reduced (transfer) admittance matrix $Y=Y^{T} \in \mathbb{C}^{n \times n}$, where $Y_{i i}$ is the self-admittance of generator $i$ and $\Re\left(Y_{i j}\right) \geq 0$ and $\Im\left(Y_{i j}\right)>0$, $i \neq j$, are the transfer conductance and (inductive) transfer susceptance between generator $i$ and $j$ in per unit values. With the power-angle relationship, the active output power $P_{\mathrm{e}, i}$ is then

$$
P_{\mathrm{e}, i}=\sum_{j=1}^{n} E_{i} E_{j}\left(\Re\left(Y_{i j}\right) \cos \left(\theta_{i}-\theta_{j}\right)+\Im\left(Y_{i j}\right) \sin \left(\theta_{i}-\theta_{j}\right)\right) .
$$

Given the transfer admittance $Y_{i j}$ between generator $i$ and $j$, define the magnitude $\left|Y_{i j}\right|>0$ and the phase shift $\varphi_{i j}=\arctan \left(\Re\left(Y_{i j}\right) / \Im\left(Y_{i j}\right)\right) \in[0, \pi / 2[$ depicting the energy loss due to the transfer conductance $\Re\left(Y_{i j}\right)$. Recall that a lossless network is characterized by zero phase shifts. Furthermore, we define the natural frequency $\omega_{i}:=P_{\mathrm{m}, i}-E_{i}^{2} \Re\left(Y_{i i}\right)$ (effective power input to generator $i$ ) and the coupling weights $P_{i j}:=E_{i} E_{j}\left|Y_{i j}\right|$ (maximum power transferred between generators $i$ and $j$ ) with $P_{i i}:=0$ for $i \in\{1, \ldots, n\}$. The network-reduced power system model can then be formulated compactly as

$$
M_{i} \ddot{\theta}_{i}=-D_{i} \dot{\theta}_{i}+\omega_{i}-\sum_{j=1}^{n} P_{i j} \sin \left(\theta_{i}-\theta_{j}+\varphi_{i j}\right) .
$$

Typically, a dynamical model for the internal voltage of generator $i$ is given as $\dot{E}_{i}=\dot{E}_{i}\left(E_{i}, u_{i}, \theta_{i}-\theta_{j}\right)$, where $u_{i}$ is the field excitation and can be used as a control input [43]. Higher order electrical and flux dynamics can be reduced [44] into an augmented damping constant $D_{i}$ in equation (3). The generator's internal excitation control essentially increases the damping torque towards the net frequency and can also be reduced into the damping constant $D_{i}$ [40], [44]. It is commonly agreed that the classical model (3) 
captures the power system dynamics sufficiently well during the first swing. Thus, we omit higher order dynamics and control effects and assume they are incorporated into the model (3). We remark that all our results are also valid if $E_{i}=E_{i}(t)$ is a smooth, bounded, and strictly positive time-varying parameter.

A frequency equilibrium of (3) is characterized by $\dot{\theta}=\mathbf{0}$ and by the (reduced) real power flow equations

$$
Q_{i}(\theta):=\omega_{i}-\sum_{j=1}^{n} P_{i j} \sin \left(\theta_{i}-\theta_{j}+\varphi_{i j}\right) \equiv 0, \quad i \in\{1, \ldots, n\} .
$$

depicting the power balance. More general, the generators are said to be in a synchronous equilibrium if all angular distances $\left|\theta_{i}-\theta_{j}\right|$ are constant and bounded (phase cohesive) and all frequencies are identical $\dot{\theta}_{i}=\dot{\theta}_{j}$. Exponential synchronization is then understood as defined before for the Kuramoto model (2).

In order to analyze the synchronization problem, system (3) is usually formulated in relative coordinates [45]. To render the resulting dynamics self-contained, uniform damping is sometimes assumed, i.e., $D_{i} / M_{i}$ is constant. Some other times, the existence of an infinite bus (a stationary generator without dynamics) as reference is postulated [4], [10]. We remark that both of these assumptions are not physically justified but are mathematical simplifications to reduce the synchronization problem to a stability analysis.

\section{B. Review of Classic Transient Stability Analysis}

Classically, transient stability analysis deals with a special case of the synchronization problem, namely the stability of a post-fault frequency equilibrium, that is, a new equilibrium of (3) arising after a change in the network parameters or topology. To answer this question various sophisticated analytic and numeric methods have been developed [10], [11], [12], [13], which typically employ the Hamiltonian structure of system (3). Since in general a Hamiltonian function for model (3) with non-trivial network conductance $\Re\left(Y_{i j}\right)>0$ (or equivalently $\varphi_{i j}>0$ ) does not exist [46], early transient stability approaches neglect the phase shifts $\varphi_{i j}$ [4], [6], [10]. In this case, the power network model (3) takes form

$$
M \ddot{\theta}=-D \dot{\theta}-\nabla U(\theta)^{T},
$$

where $\nabla$ is the gradient and $U:]-\pi, \pi]^{n} \rightarrow \mathbb{R}$ is the potential energy given up to an additive constant by

$$
U(\theta)=-\sum_{i=1}^{n}\left(\omega_{i} \theta_{i}+\sum_{j=1}^{n} P_{i j}\left(1-\cos \left(\theta_{i}-\theta_{j}\right)\right)\right) .
$$

When system (5) is formulated in relative or reference coordinates (that feature equilibria), the energy function $(\theta, \dot{\theta}) \mapsto(1 / 2) \dot{\theta}^{T} M \dot{\theta}+U(\theta)$ serves (locally) as a Lyapunov function. In combination with the invariance principle, we clearly have that the dynamics (5) converge to $\dot{\theta}=\mathbf{0}$ and the largest invariant zero level set of $\nabla U(\theta)$. In order to estimate the region of attraction of a stable equilibrium, algorithms such as PEBS [4] or $B C U$ [7] consider the associated dimension-reduced gradient flow

$$
\dot{\theta}=-\nabla U(\theta)^{T} .
$$

Then $\left(\theta^{*}, \mathbf{0}\right)$ is a hyperbolic type- $k$ equilibrium of $(5)$, i.e., the Jacobian has $k$ stable eigenvalues, if and only if $\theta^{*}$ is a hyperbolic type- $k$ equilibrium of (7), and if a generic transversality condition holds, then 
the regions of attractions of both equilibria are bounded by the stable manifolds of the same unstable equilibria [4, Theorems 6.2-6.3]. This topological equivalence between (5) and (7) can also be extended to "sufficiently small" transfer conductances [7, Theorem 5.7]. For further interesting relationships among the systems (5) and (7), we refer to [4], [7], [12], [10]. Other approaches to lossy power networks with non-zero transfer conductances compute numerical energy functions [5] or employ an extended invariance principle [9]. Based on these results computational methods were developed to approximate the stability boundaries of (5) and (7) by level sets of energy functions or stable manifolds of unstable equilibria.

To summarize the shortcomings of the classical transient stability analysis methods, they consider simplified models formulated in reference or relative coordinates (with uniform damping assumption) and result mostly in numerical procedures rather than in concise and simple conditions. For lossy power networks the cited articles consider either special benchmark problems or networks with "sufficiently small" transfer conductances. To the best of our knowledge there are no results quantifying this smallness of $\Re\left(Y_{i j}\right)$ or $\varphi_{i j}$ for arbitrary networks. Moreover, from a network perspective the existing methods do not result in explicit and concise conditions relating synchronization to the network's state, parameters, and topology. The following sections will address these questions quantitatively via purely algebraic tests.

\section{The Non-Uniform Kuramoto Model And Main Synchronization Result}

\section{A. The Non-Uniform Kuramoto Model}

As we have already mentioned, there is a striking similarity between the power network model (3) and the Kuramoto model (2). To study this similarity, we define the non-uniform Kuramoto model by

$$
D_{i} \dot{\theta}_{i}=\omega_{i}-\sum_{j=1}^{n} P_{i j} \sin \left(\theta_{i}-\theta_{j}+\varphi_{i j}\right), \quad i \in\{1, \ldots, n\},
$$

where we assume that the parameters satisfy $D_{i}>0, \omega_{i} \in \mathbb{R}, P_{i j}>0$, and $\varphi_{i j} \in[0, \pi / 2[$, for all $i, j \in\{1, \ldots, n\}, i \neq j$; by convention, $P_{i i}$ and $\varphi_{i i}$ are set to zero. System (8) may be regarded as a generalization of the classic Kuramoto model (2) with multiple time-constants $D_{i}$ and non-homogeneous but symmetric coupling terms $P_{i j}$ and phase shifts $\varphi_{i j}$. The non-uniform Kuramoto model (8) will serve as a link between the power network model (3), the Kuramoto model (2), and the consensus protocol (1).

Remark III.1 (Second-order systems and their first-order approximations:) The non-uniform Kuramoto model (8) can be seen as a long-time approximation of the second order system (3) for a small "inertia over damping ratio" $M_{i} / D_{i}$. Note the analogy between the non-uniform Kuramoto model (8) and the dimension-reduced gradient system (7) studied in classic transient stability analysis to approximate the stability properties of the second-order system (5) [4], [7], [10]. Both models are of first order, have the same right-hand side, and differ only in the time constants $D_{i}$. Thus, both models have the same equilibria with the same stability properties and with regions of attractions bounded by the same separatrices [4, Theorems 3.1-3.4]. The reduced system (7) is formulated as a gradient-system to study the stability of 
the equilibria of (7) (possibly in relative coordinates). The non-uniform Kuramoto model (8), on the other hand, can be directly used to study synchronization and reveals the underlying network structure.

\section{B. Main Synchronization Result}

We can now state our main result on the power network model (3) and the non-uniform Kuramoto model (8).

Theorem III.2 (Main synchronization result) Consider the power network model (3) and the nonuniform Kuramoto model (8). Assume that the minimal lossless coupling of any oscillator to the network is larger than a critical value, i.e.,

$\Gamma_{\min }:=n \min _{i \neq j}\left\{\frac{P_{i j}}{D_{i}} \cos \left(\varphi_{i j}\right)\right\}>\Gamma_{\text {critical }}:=\frac{1}{\cos \left(\varphi_{\max }\right)}\left(\max _{i \neq j}\left|\frac{\omega_{i}}{D_{i}}-\frac{\omega_{j}}{D_{j}}\right|+2 \max _{i \in\{1, \ldots, n\}} \sum_{j=1}^{n} \frac{P_{i j}}{D_{i}} \sin \left(\varphi_{i j}\right)\right)$.

Accordingly, define $\gamma_{\min } \in\left[0, \pi / 2-\varphi_{\max }\left[\right.\right.$ and $\left.\left.\gamma_{\max } \in\right] \pi / 2, \pi\right]$ as unique solutions to the equations $\sin \left(\gamma_{\min }\right)=\sin \left(\gamma_{\max }\right)=\cos \left(\varphi_{\max }\right) \Gamma_{\text {critical }} / \Gamma_{\min }$.

For the non-uniform Kuramoto model,

1) phase cohesiveness: the set $\bar{\Delta}(\gamma)$ is positively invariant for every $\gamma \in\left[\gamma_{\min }, \gamma_{\max }\right]$, and each trajectory starting in $\Delta\left(\gamma_{\max }\right)$ reaches $\bar{\Delta}\left(\gamma_{\min }\right)$; and

2) frequency synchronization: for every $\theta(0) \in \Delta\left(\gamma_{\max }\right)$, the frequencies $\dot{\theta}_{i}(t)$ synchronize exponentially to some frequency $\dot{\theta}_{\infty} \in\left[\dot{\theta}_{\min }(0), \dot{\theta}_{\max }(0)\right]$.

For the power network model, for all $\theta(0) \in \Delta\left(\gamma_{\max }\right)$ and initial frequencies $\dot{\theta}_{i}(0)$,

3) approximation error: there exists a constant $\epsilon^{*}>0$ such that, if $\epsilon:=M_{\max } / D_{\min }<\epsilon^{*}$, then the solution $(\theta(t), \dot{\theta}(t))$ of (3) exists for all $t \geq 0$ and it holds uniformly in $t$ that

$$
\begin{aligned}
\left(\theta_{i}(t)-\theta_{n}(t)\right) & =\left(\bar{\theta}_{i}(t)-\bar{\theta}_{n}(t)\right)+\mathcal{O}(\epsilon), \quad \forall t \geq 0, i \in\{1, \ldots, n-1\}, \\
\dot{\theta}(t) & =D^{-1} Q(\bar{\theta}(t))+\mathcal{O}(\epsilon), \quad \forall t>0,
\end{aligned}
$$

where $\bar{\theta}(t)$ is the solution to the non-uniform Kuramoto model (8) with initial condition $\bar{\theta}(0)=\theta(0)$, and $D^{-1} Q(\bar{\theta})$ is the power flow (4) scaled by the inverse damping $D^{-1}$; and

4) asymptotic approximation error: there exists $\epsilon$ and $\varphi_{\max }$ sufficiently small, such that the $\mathcal{O}(\epsilon)$ approximation errors in equation (10) converge to zero as $t \rightarrow \infty$.

The proof of Theorem III.2 is based on a singular perturbation analysis of the power network model (3) (see Section IV) and a synchronization analysis of the non-uniform Kuramoto model (8) (see Section V) and will be postponed to the end of Section V. We discuss the assumption that the perturbation parameter $\epsilon$ needs to be small separately in the next subsection and state the following remarks to Theorem III.2:

Remark III.3 (Physical interpretation of Theorem III.2:) The right-hand side of condition (9) states the worst-case non-uniformity in natural frequencies (the difference in effective power inputs at each 
generator) and the worst-case lossy coupling of a generator to the network $\left(P_{i j} \sin \left(\varphi_{i j}\right)=E_{i} E_{j} \Re\left(Y_{i j}\right)\right.$ reflects the transfer conductance), both of which are scaled with the rates $D_{i}$. The term $\cos \left(\varphi_{\max }\right)=$ $\sin \left(\pi / 2-\varphi_{\max }\right)$ corresponds to phase cohesiveness in $\Delta\left(\pi / 2-\varphi_{\max }\right)$, which is necessary for the latter consensus-type analysis. These negative effects have to be dominated by the left-hand side of (9), which is a lower bound for $\min _{i}\left\{\sum_{j=1}^{n}\left(P_{i j} \cos \left(\varphi_{i j}\right) / D_{i}\right)\right\}$, the worst-case lossless coupling of a node to the network. The multiplicative gap $\Gamma_{\text {critical }} / \Gamma_{\text {min }}$ between the right- and the left-hand side in (9) can be understood as a robustness margin that additionally gives a practical stability result determining the admissible initial and the possible ultimate lack of phase cohesiveness in $\bar{\Delta}\left(\gamma_{\min }\right)$ and $\bar{\Delta}\left(\gamma_{\max }\right)$.

In summary, the conditions of Theorem III.2 read as "the network connectivity has to dominate the network's non-uniformity, the network's losses, and the lack of phase cohesiveness." In Theorem III.2 we present the scalar synchronization condition (9), the estimate for the region of attraction $\Delta\left(\gamma_{\max }\right)$, and the ultimate phase cohesive set $\bar{\Delta}\left(\gamma_{\min }\right)$. In the derivations leading to Theorem III.2 it is possible to trade off a tighter synchronization condition against a looser estimate of the region of attraction, or a single loose scalar condition against $n(n-1) / 2$ tight pairwise conditions. These tradeoffs are explored in the appendix of this document. Finally, we remark that the coupling weights $P_{i j}$ in condition (9) are not only the reduced power flows but reflect for uniform voltages $E_{i}$ and phase shifts $\varphi_{i j}$ also the effective resistance of the original (non-reduced) network topology [42]. Moreover, condition (9) indicates at which generator the damping torque has to be increased or decreased (via local power system stabilizers) in order to meet the sufficient synchronization conditions.

The power network model (3) inherits the synchronization condition (9) in the relative coordinates $\theta_{i}-\theta_{n}$ and up to the approximation error (10) which is of order $\epsilon$ and eventually vanishes for $\epsilon$ and $\varphi_{\max }$ sufficiently small. The relative coordinates can be shown to be well-posed (see Section IV). The convergence of the power network model only from almost all initial conditions is a consequence of the existence of saddle points in the non-uniform Kuramoto model.

Remark III.4 (Refinement of Theorem III.2:) Theorem III.2 can also be stated for two-norm bounds on the parameters involving the algebraic connectivity (see Theorem V.5). For a lossless network, explicit values for the synchronization frequency and the exponential synchronization rate as well as conditions for phase synchronization can be derived (see Theorems V.1 and V.10). When specialized to the classic Kuramoto model (2), the sufficient condition (9) is improves the results [24], [25], [32], [33], [39], and it can also shown to be a tight bound. We refer the reader to the detailed comments in Section V.

\section{Discussion of the Perturbation Assumption}

The assumption that each generator is strongly overdamped is captured by the smallness of the perturbation parameter $\epsilon=M_{\max } / D_{\min }$. This choice of the perturbation parameter and the subsequent singular perturbation analysis (in Section IV) is similar to the analysis of Josephson arrays [26], coupled overdamped mechanical pendula [47], flocking models [48], and also classic transient stability analysis 
[4, Theorem 5.2], [36]. In the linear case, this analysis resembles the well-known overdamped harmonic oscillator, which features one slow and one fast eigenvalue. The overdamped harmonic oscillator exhibits two time-scales and the fast eigenvalue corresponding to the frequency damping can be neglected in the long-term phase dynamics. In the non-linear case these two distinct time-scales are captured by a singular perturbation analysis. In short, this reduction of a coupled-pendula system corresponds to the assumption that damping to a synchronization manifold and synchronization itself occur on separate time scales.

In the application to realistic generator models one has to be careful under which operating conditions $\epsilon$ is indeed a small physical quantity. Typically, $M_{i} \in[2 \mathrm{~s}, 12 \mathrm{~s}] /\left(2 \pi f_{0}\right)$ depending on the type of generator and the mechanical damping (including damper winding torques) is poor: $D_{i} \in[1,3] /\left(2 \pi f_{0}\right)$. However, for the synchronization problem also the generator's internal excitation control have to be considered, which increases the damping torque to $D_{i} \in[10,35] /\left(2 \pi f_{0}\right)$ depending on the system load [41], [40], [44]. In this case, $\epsilon \in \mathcal{O}(0.1)$ is indeed a small quantity and a singular perturbation approximation is accurate. In fact, the recent power systems literature discusses the need for sufficiently large damping to enhance transient stability, see [49], [50] and references therein.

We note that simulation studies show an accurate approximation of the power network by the nonuniform Kuramoto model also for values of $\epsilon \in \mathcal{O}(1)$, i.e., they indicate that the threshold $\epsilon^{*}$ may be sizable. The theoretical reasoning is the topological equivalence discussed in Subsection II-B between the power network model (3) and the first-order model (7), which is again topologically equivalent to the non-uniform Kuramoto model (8), as discussed in Remark III.1. The synchronization condition (9) on the non-uniform Kuramoto model (8) guarantees exponential stability of the non-uniform Kuramoto dynamics formulated in relative coordinates $\theta_{i}-\theta_{n}$, which again implies local exponential stability of the power network model (3) in relative coordinates. These arguments are elaborated in detail in the next section. Thus, from the viewpoint of topological equivalence, Theorem III.2 holds locally completely independent of $\epsilon>0$, and the magnitude of $\epsilon$ gives a bound on the approximation errors (10) during transients.

The analogies between the power network model (3) and the reduced model (7), corresponding to the non-uniform Kuramoto model (8), are directly employed in the PEBS [4] and $B C U$ algorithms [7]. These algorithms are not only scholastic but applied by the power industry [38], which additionally supports the validity of the approximation of the power network model by the non-uniform Kuramoto model. 


\section{Singular Perturbation Analysis of Synchronization}

\section{A. Time-Scale Separation of the Power Network Model}

In this section, we put the approximation of the power network model (3) by the non-uniform Kuramoto model (8) on solid mathematical ground via a singular perturbation analysis. The analysis by Tikhonov's method [51] requires a system evolving on Euclidean space and exponentially stable fixed points. In order to satisfy the assumptions of Tikhonov's theorem, we introduce two concepts.

First, we introduce a smooth map from a suitable subset of $\mathbb{T}^{n}$ to a compact subset of $\mathbb{R}^{n-1}$. For $\gamma \in\left[0, \pi\left[\right.\right.$, define the map grnd $: \Delta(\gamma) \rightarrow \Delta_{\text {grnd }}(\gamma):=\left\{\bar{\delta} \in \mathbb{R}^{n-1}|| \bar{\delta}_{i}\left|<\gamma, \max _{i, j}\right| \bar{\delta}_{i}-\bar{\delta}_{j} \mid<\gamma, i, j \in\right.$ $\{1, \ldots, n-1\}\}$ that associates to the angles $\left(\theta_{1}, \ldots, \theta_{n}\right) \in \Delta(\gamma)$ the array of angle differences $\bar{\delta}$ with components $\bar{\delta}_{i}=\theta_{i}-\theta_{n}$, for $i \in\{1, \ldots, n-1\}$. This map is well defined, that is, $\bar{\delta} \in \Delta_{\text {grnd }}(\gamma)$, because $\left|\bar{\delta}_{i}\right|=\left|\theta_{i}-\theta_{n}\right|<\gamma$ for all $i \in\{1, \ldots, n-1\}$ and $\left|\bar{\delta}_{i}-\bar{\delta}_{j}\right|=\left|\theta_{i}-\theta_{j}\right|<\gamma$ for all distinct $i, j \in\{1, \ldots, n-1\}$. Also, this map is smooth because $\gamma<\pi$ implies that all angles take value in an open half-circle and their pairwise differences are smooth functions (see Section I). As a final remark,

note that the angle differences $\bar{\delta}_{1}, \ldots, \bar{\delta}_{n-1}$ are well-known in the transient stability [7], [52] and in the Kuramoto literature [29], and we refer to them as grounded angles in the spirit of circuit theory. The sets $\Delta(\pi)$ and $\Delta_{\text {grnd }}(\pi)$ as well as the map $\theta \mapsto \bar{\delta}=\operatorname{grnd}(\theta)$ are illustrated in Figure 1.

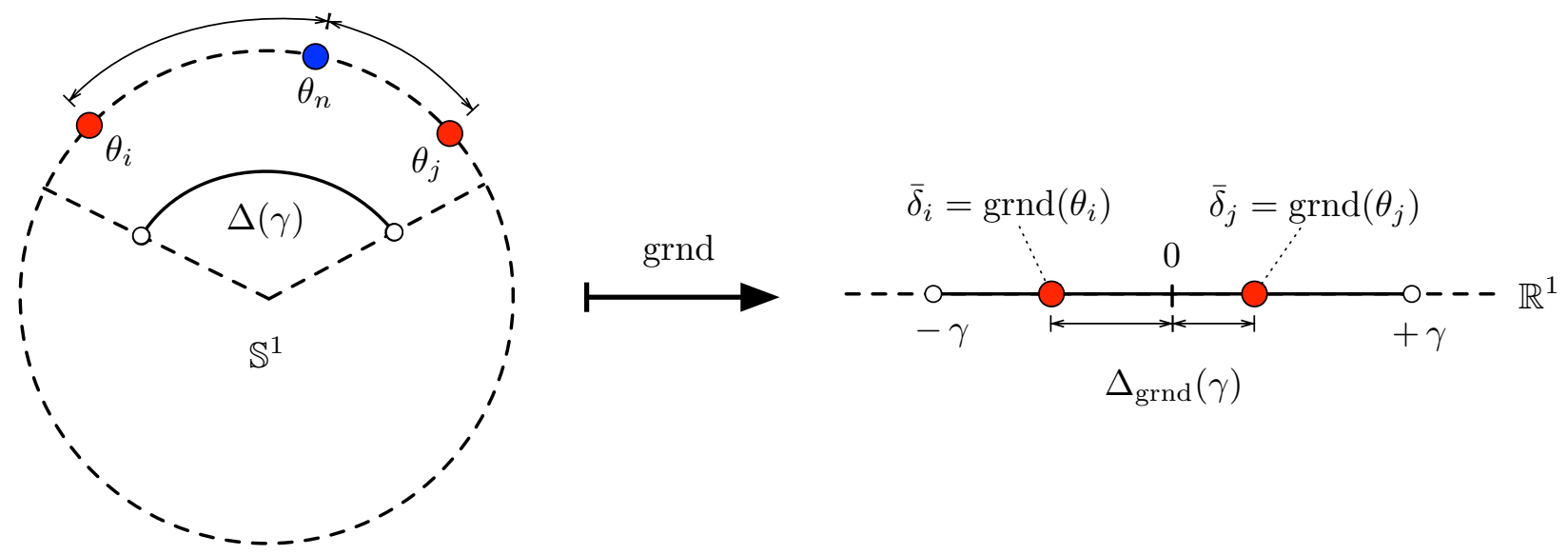

Fig. 1. Illustration of the map grnd : $\Delta(\gamma) \rightarrow \Delta_{\text {grnd }}(\gamma)$. The map grnd can be thought of as as a symmetry-reducing projection from $\Delta(\gamma)$ (illustrated as subset of $\mathbb{S}^{1}$ ) to $\Delta_{\text {grnd }}(\gamma)$ (illustrated as subset of $\mathbb{R}^{1}$ ), where $\theta_{n}$ is projected to the origin 0 . The set $\Delta(\gamma)$ and the map grnd are invariant under translations on $\mathbb{T}^{n}$ that is, under maps of the form $\left(\theta_{1}, \ldots, \theta_{n}\right) \mapsto\left(\theta_{1}+\alpha, \ldots \theta_{n}+\alpha\right)$.

Second, by formally computing the difference between the angles $\dot{\theta}_{i}$ and $\dot{\theta}_{n}$, we define grounded Kuramoto model with state $\delta \in \mathbb{R}^{n-1}$ by

$\dot{\delta}_{i}=\frac{\omega_{i}}{D_{i}}-\frac{\omega_{n}}{D_{n}}-\sum_{j=1, j \neq i}^{n-1}\left(\frac{P_{i j}}{D_{i}} \sin \left(\delta_{i}-\delta_{j}+\varphi_{i j}\right)+\frac{P_{n j}}{D_{n}} \sin \left(\delta_{j}-\varphi_{j n}\right)\right)-\left(\frac{P_{i n}}{D_{i}} \sin \left(\delta_{i}+\varphi_{i n}\right)+\frac{P_{i n}}{D_{n}} \sin \left(\delta_{i}-\varphi_{i n}\right)\right)$. 
Lemma IV.1 (Properties of grounded Kuramoto model) Let $\gamma \in\left[0, \pi\left[\right.\right.$ and let $\theta: \mathbb{R}_{\geq 0} \rightarrow \mathbb{T}^{n}$ be a solution to the non-uniform Kuramoto model (8) satisfying $\theta(0) \in \Delta(\gamma)$. Let $\delta: \mathbb{R}_{\geq 0} \rightarrow \mathbb{R}^{n-1}$ be the solution to the grounded Kuramoto model (11) with initial condition $\delta(0)=\operatorname{grnd}(\theta(0)) \in \Delta_{\text {grnd }}(\gamma)$.

Then, $\delta(t)=\operatorname{grnd}(\theta(t))$ for all $t \geq 0$, if any one of the two following equivalent conditions holds:

1) phase cohesiveness: the angles $\theta(t)$ take value in $\Delta(\gamma)$ for all time $t \geq 0$; and

2) well-posedness: the grounded angles $\delta(t)$ take value in $\Delta_{\text {grnd }}(\gamma)$ for all time $t \geq 0$.

Moreover, the following two statements are equivalent for any $\gamma \in[0, \pi[$ :

3) exponential frequency synchronization: each trajectory of the non-uniform Kuramoto model satisfying the phase cohesiveness property 1) achieves exponential frequency synchronization; and

4) exponential convergence to equilibria: each trajectory of the grounded Kuramoto model satisfying the well-posedness property 2) converges exponentially to an equilibrium point.

Finally, every trajectory of the grounded Kuramoto model as in 4) satisfying the well-posedness property 2) with $\gamma \in\left[0, \pi / 2-\varphi_{\max }\right]$ converges to an exponentially stable equilibrium point.

Proof: Note that, since both vector fields (8) and (11) are locally Lipschitz, existence and uniqueness of the corresponding solutions follows provided that the corresponding evolutions are compact. Now, assume that 1) holds, that is, $\theta(t) \in \Delta(\gamma)$ (compact) for all $t \geq 0$. Therefore, $\bar{\delta}(t)=\operatorname{grnd}(\theta(t)) \in$ $\Delta_{\text {grnd }}(\gamma)$ for all $t \geq 0$. Also recall that the map grnd is smooth over $\Delta(\gamma)$. These facts and the definition of the grounded Kuramoto model (11) imply that $\frac{d}{d t} \operatorname{grnd}(\theta(t))$ is well defined and identical to $\dot{\delta}(t)$ for all $t \geq 0$. In turn, this implies that $\delta(t)=\operatorname{grnd}(\theta(t)) \in \Delta_{\text {grnd }}(\gamma)$ holds for all positive times.

Conversely, assume that 2) holds, that is, $\delta(t) \in \Delta_{\text {grnd }}(\gamma)$ (compact) for all $t \geq 0$. Due to existence and uniqueness and since initially $\delta(0)=\operatorname{grnd}(\theta(0))$ with $\theta(0) \in \Delta(\gamma)$, a set of angles $\theta(t) \in \Delta(\gamma)$ can be associated to $\delta(t) \in \Delta_{\text {grnd }}(\gamma)$ such that $\delta(t)=\operatorname{grnd}(\theta(t))$ for all $t \geq 0$. By construction of the grounded Kuramoto model (11), we have that $\theta(t)$ is identical to the solution to the non-uniform Kuramoto model (8) for all $t \geq 0$. Thus, statement 2) implies statement 1) and $\delta(t)=\operatorname{grnd}(\theta(t))$ for all $t \geq 0$. Having established the equivalence of 1) and 2), we do not further distinguish between $\delta(t)$ and $\operatorname{grnd}(\theta(t))$.

Assume that 3 ) holds, that is, all $\dot{\theta}_{i}(t)$ converge exponentially fast to some $\dot{\theta}_{\infty} \in \mathbb{R}$. It follows that each $\dot{\delta}_{i}(t)=\dot{\theta}_{i}(t)-\dot{\theta}_{n}(t)$ converges exponentially fast to zero, and $\delta(t)=\delta(0)+\int_{0}^{t} \dot{\delta}(\tau) d \tau$ converges exponentially fast to some $\delta_{\infty} \in \Delta_{\text {grnd }}(\gamma)$ due to property 2). Since the vector field (11) is continuous and $\lim _{t \rightarrow \infty}(\delta(t), \dot{\delta}(t))=\left(\delta_{\infty}, \mathbf{0}\right)$, the vector $\delta_{\infty}$ is necessarily an equilibrium of (11), and property 4) follows.

Assume that 4$)$ holds, that is, all angular differences $\delta_{i}(t)=\theta_{i}(t)-\theta_{n}(t)$ converge exponentially fast to constant values $\delta_{i, \infty}$ for $i \in\{1, \ldots, n-1\}$. This fact and the continuity of the vector field in equation (11) imply that the array with entries $\delta_{i, \infty}$ is an equilibrium for (11) and that each frequency difference $\dot{\delta}_{i}(t)=\dot{\theta}_{i}(t)-\dot{\theta}_{n}(t)$ converges to zero. Moreover, because the vector field in equation (11) is analytic and the solution converges exponentially fast to an equilibrium point, the right-hand side of equation (11) converges exponentially fast to zero and thus also the time-derivative of the solution, i.e., the array of all frequency differences, converges exponentially fast to zero. 
To prove the final statement, assume that the non-uniform Kuramoto model (8) achieves frequency synchronization with synchronization frequency $\dot{\theta}_{\text {sync }} \in \mathbb{R}^{1}$ and phase cohesiveness in $\Delta\left(\pi / 2-\varphi_{\max }\right)$. Thus, when formulated in a rotating coordinate frame with zero synchronization frequency, all trajectories $\theta_{i}(t)-\dot{\theta}_{\text {sync }} \cdot t(\bmod 2 \pi)$ necessarily converge to an equilibrium $\theta^{*} \in \Delta\left(\pi / 2-\varphi_{\max }\right)$.

The Jacobian $J\left(\theta^{*}\right)$ of the non-uniform Kuramoto model is given by the Laplacian matrix with weights $a_{i j}\left(\theta^{*}\right)=\left(P_{i j} / D_{i}\right) \cos \left(\theta_{i}^{*}-\theta_{j}^{*}+\varphi_{i j}\right)$. For any $\theta^{*} \in \Delta\left(\pi / 2-\varphi_{\max }\right)$, the weights $a_{i j}\left(\theta^{*}\right)$ are strictly positive. In this case, it follows from the contraction property [53, Theorem 1] that the linearized dynamics $\dot{\theta}=J\left(\theta^{*}\right) \cdot \theta$ converge from any initial condition in $\mathbb{R}^{n}$ to a point in the diagonal vector space $\mathbf{1}_{n}$. Hence, for any $\theta^{*} \in \Delta\left(\pi / 2-\varphi_{\max }\right)$, the matrix $J\left(\theta^{*}\right)$ has $n-1$ stable eigenvalues and one zero eigenvalue with eigenspace $\mathbf{1}_{n}$ corresponding to the translational invariance of the angular variable.

Hence, any equilibrium manifold $\theta^{*} \in \Delta\left(\pi / 2-\varphi_{\max }\right)$ (of dimension one due to translational invariance) is exponentially stable w.r.t. to the non-uniform Kuramoto dynamics (8). The corresponding point $\delta^{*}=$ $\operatorname{grnd}\left(\theta^{*}(t)\right) \in \Delta_{\text {grnd }}\left(\pi / 2-\varphi_{\max }\right)$ (the translational symmetry is removed) is an equilibrium of the grounded Kuramoto dynamics (11) (due to property 4)). Finally, since $\theta^{*}$ is exponentially stable, it necessarily follows that $\delta^{*}$ is an exponentially stable equilibrium point.

As mentioned in Remark III.1, system (8) may be seen a long-time approximation of (3), or spoken differently, it is the reduced system obtained by a singular perturbation analysis. A physically reasonable singular perturbation parameter is the worst-case choice of $M_{i} / D_{i}$, that is, $\epsilon:=M_{\max } / D_{\min }$. The dimension of $\epsilon$ is in seconds, which makes sense since time still has to be normalized with respect to $\epsilon$. If we reformulate the power network model (3) in grounded angular coordinates with the state $(\delta, \dot{\theta}) \in \mathbb{R}^{n-1} \times \mathbb{R}^{n}$, then we obtain the following system in singular perturbation standard form

$$
\begin{aligned}
\frac{d}{d t} \delta_{i} & =f_{i}(\dot{\theta}):=\dot{\theta}_{i}-\dot{\theta}_{n}, \quad i \in\{1, \ldots, n-1\}, \\
\epsilon \frac{d}{d t} \dot{\theta}_{i} & =g_{i}(\delta, \dot{\theta}):=-F_{i} \dot{\theta}_{i}+\frac{F_{i}}{D_{i}}\left(\omega_{i}-\sum_{j=1}^{n} P_{i j} \sin \left(\delta_{i}-\delta_{j}+\varphi_{i j}\right)\right), \quad i \in\{1, \ldots, n\},
\end{aligned}
$$

where $F_{i}:=\left(D_{i} / D_{\min }\right) /\left(M_{i} / M_{\max }\right)$ and $\delta_{n}:=0$ in equation (13). For $\epsilon$ sufficiently small, the long-term dynamics of (12)-(13) can be approximated by the grounded Kuramoto model (11) and the power flow (4), where the approximation error is of order $\epsilon$ and $F_{i}$ determines its convergence rate in the fast time-scale.

Theorem IV.2 (Singular Perturbation Approximation) Consider the power network model (3) written as the singular perturbation problem (12)-(13) with bounded initial conditions $(\delta(0), \dot{\theta}(0))$, and the grounded non-uniform Kuramoto model (11) with initial condition $\delta(0)$ and solution $\bar{\delta}(t)$. Assume that there exists an exponentially stable fixed point $\delta_{\infty}$ of $(11)$ and $\delta(0)$ is in a compact subset $\Omega_{\delta}$ of its region of attraction. Then, for each $\Omega_{\delta}$

1) there exists $\epsilon_{*}>0$ such that for all $\epsilon<\epsilon_{*}$, the singular perturbation problem (12)-(13) has a unique solution $(\delta(t, \epsilon), \dot{\theta}(t, \epsilon))$ for $t \geq 0$, and for all $t \geq 0$ it holds uniformly in $t$ that

$$
\delta(t, \epsilon)-\bar{\delta}(t)=\mathcal{O}(\epsilon), \quad \text { and } \quad \dot{\theta}(t, \epsilon)-h(\bar{\delta}(t))-y(t / \epsilon)=\mathcal{O}(\epsilon),
$$


where $y_{i}(t / \epsilon):=\left(\dot{\theta}_{i}(0)-h_{i}(\delta(0))\right) e^{-F_{i} t / \epsilon}$ and $h_{i}(\delta):=Q_{i}(\delta) / D_{i}$ for $i \in\{1, \ldots, n\}$.

2) For any $t_{b}>0$, there exists $\epsilon^{*} \leq \epsilon_{*}$ such that for all $t \geq t_{b}$ and whenever $\epsilon<\epsilon^{*}$ it holds uniformly that

$$
\dot{\theta}(t, \epsilon)-h(\bar{\delta}(t))=\mathcal{O}(\epsilon) .
$$

3) Additionally, there exist $\epsilon$ and $\varphi_{\max }$ sufficiently small such that the approximation errors (14)-(15) converge exponentially to zero as $t \rightarrow \infty$.

Proof: To prove statements 1) and 2) of Theorem IV.2 we will follow Tikhonov's theorem [51, Theorem 11.2] and show that the singularly perturbed system (12)-(13) satisfies all assumptions of [51, Theorem 11.2] when analyzing it on $\mathbb{R}^{n-1} \times \mathbb{R}^{n}$ and after translating the arising fixed point to the origin.

Exponential stability of the reduced system: The quasi-steady-state of (12)-(13) is obtained by solving $g_{i}(\delta, \dot{\theta})=0$ for $\dot{\theta}$, resulting in the unique (and thus isolated) root $\dot{\theta}_{i}=h_{i}(\delta)=Q_{i}(\delta) / D_{i}, i \in\{1, \ldots, n\}$. The reduced system is obtained as $\dot{\delta}_{i}=f_{i}(h(\delta))=h_{i}(\delta)-h_{n}(\delta), i \in\{1, \ldots, n-1\}$, which is equivalent to the grounded non-uniform Kuramoto model (11). The reduced system is smooth, evolves on $\mathbb{R}^{n-1}$, and by assumption its solution $\bar{\delta}(t)$ is bounded and converges exponentially to the stable fixed point $\delta_{\infty}$. Define the error coordinates $x_{i}(t)=\bar{\delta}_{i}(t)-\delta_{i, \infty}, i \in\{1, \ldots, n-1\}$ and the resulting system $\dot{x}=f\left(h\left(x+\delta_{\infty}\right)\right)$ with state in $\mathbb{R}^{n-1}$ and initial condition $x(0)=\delta(0)-\delta_{\infty}$. By assumption, the solution $x(t)$ is bounded and converges exponentially to the stable fixed point at $x=\mathbf{0}$.

Exponential stability of the boundary layer system: Consider the error coordinate $y_{i}=\dot{\theta}_{i}-h_{i}(\delta)$, which shifts the error made by the quasi-stationarity assumption $\dot{\theta}_{i}(t) \approx h_{i}(\delta(t))$ to the origin. After stretching time to the dimensionless variable $\tau=t / \epsilon$, the quasi-steady-state error is

$$
\frac{d}{d \tau} y_{i}=g_{i}(\delta, y+h(\delta))-\epsilon \frac{\partial h_{i}}{\partial \delta} f(y+h(\delta))=-F_{i} y_{i}-\epsilon \frac{\partial h}{\partial \delta} f_{i}(y+h(\delta))
$$

with $y_{i}(0)=\dot{\theta}_{i}(0)-h_{i}(\delta(0))$. By setting $\epsilon=0,(16)$ reduces to the boundary layer model

$$
\frac{d}{d \tau} y_{i}=-F y_{i}, \quad y_{i}(0)=\dot{\theta}_{i}(0)-h_{i}(\delta(0)) .
$$

The boundary layer model (17) is globally exponentially stable with solution $y_{i}(t / \epsilon)=y_{i}(0) e^{-F_{i} t / \epsilon}$, where $y_{i}(0)$ is in a compact subset of the region of attraction of (17) due to boundedness of $(\delta(0), \dot{\theta}(0))$.

In summary, the singularly perturbed system (12)-(13) is smooth on $\mathbb{R}^{n-1} \times \mathbb{R}^{n}$, and the origins of the reduced system (in error coordinates) $\dot{x}=f\left(h\left(x+\delta_{\infty}\right)\right)$ and the boundary layer model (17) are exponentially stable (where Lyapunov functions are readily existent by converse arguments [51, Theorem 4.14]). Thus, all assumptions of [51, Theorem 11.2] are satisfied and statements 1) and 2) follow.

To prove statement 3 ), note that $\bar{\delta}(t)$ converges to an exponentially stable equilibrium point $\delta_{\infty}$, and $(\delta(t, \epsilon), \dot{\theta}(t, \epsilon))$ converges to an $\mathcal{O}(\epsilon)$ neighborhood of $\left(\delta_{\infty}, h\left(\bar{\delta}_{\infty}\right)\right)$, where $h\left(\bar{\delta}_{\infty}\right)=\mathbf{0}$. We now invoke classic topological equivalence arguments from the transient stability literature [4], [7]. Both the second order system (12)-(13) as well as the reduced system $\dot{\delta}=f(h(\delta))$ correspond to the perturbed Hamiltonian system (8)-(9) in [7] and the perturbed gradient system (10) in [7], where the latter is considered in [7] with $D_{i}=1$ for all $i$. Consider for a moment the case when all $\varphi_{i j}=0$. In this case, the reduced system 
has a locally exponentially stable fixed point $\delta_{\infty}$ (for any $D_{i}>0$ due to [4, Theorem 3.1]), and by [7, Theorem 5.1] we conclude that $\left(\delta_{\infty}, \mathbf{0}\right)$ is also a locally exponentially stable fixed point of the second order system (12)-(13). Furthermore, due to structural stability [7, Theorem 5.7, R1], this conclusion holds also for sufficiently small phase shifts $\varphi_{i j}$. Thus, for sufficiently small $\epsilon$ and $\varphi_{\max }$, the solution of (12)-(13) converges exponentially to $\left(\delta_{\infty}, \mathbf{0}\right)$. Consequently, the approximation errors $\delta(t, \epsilon)-\bar{\delta}(t)$ and $\dot{\theta}(t, \epsilon)-h(\bar{\delta})$ as well as the boundary layer error $y(t / \epsilon)$ converge exponentially to zero.

Theorem IV.2 can be interpreted geometrically as follows. The frequency dynamics of system (3) happen on a fast time-scale and converge exponentially to a slow manifold which can be approximated to first order by the scaled power flow $D^{-1} Q(\theta)$. On this slow manifold the long-term phase synchronization dynamics of system (3) are given by the non-uniform Kuramoto model (8).

\section{SyNCHRONIZATION OF NON-UNIFORM KURAMOTO OSCILLATORS}

This section combines and extends methods from the consensus and Kuramoto literature to analyze the non-uniform Kuramoto model (8). The role of the time constants $D_{i}$ and the phase shifts $\varphi_{i j}$ is immediately revealed when dividing by $D_{i}$ both hand sides of (8) and expanding the right-hand side as

$$
\dot{\theta}_{i}=\frac{\omega_{i}}{D_{i}}-\sum_{j=1, j \neq i}^{n}\left(\frac{P_{i j}}{D_{i}} \cos \left(\varphi_{i j}\right) \sin \left(\theta_{i}-\theta_{j}\right)+\frac{P_{i j}}{D_{i}} \sin \left(\varphi_{i j}\right) \cos \left(\theta_{i}-\theta_{j}\right)\right) .
$$

The difficulties in the analysis of system (8) are the phase shift-induced lossy coupling $\left(P_{i j} / D_{i}\right) \sin \left(\varphi_{i j}\right)$ $\times \cos \left(\theta_{i}-\theta_{j}\right)$ inhibiting synchronization and the non-symmetric coupling between an oscillator pair $\{i, j\}$ via $P_{i j} / D_{i}$ on the one hand and $P_{i j} / D_{j}$ on the other. Since the non-uniform Kuramoto model (8) is derived from the power network model (3), the underlying graph induced by $P$ is complete and symmetric, i.e., except for the diagonal entries, the matrix $P$ is fully populated and symmetric. For the sake of generality, this section considers the non-uniform Kuramoto model (8) under the assumption that the graph induced by $P$ is neither complete nor symmetric, that is, some $P_{i j}$ are zero and $P \neq P^{T}$.

\section{A. Frequency Synchronization of Phase-Cohesive Oscillators}

Under the assumption of cohesive phases, the classic Kuramoto model (2) achieves frequency synchronization [32, Theorem 3.1], [39, Corollary 11]. An analogous result guarantees frequency synchronization of non-uniform Kuramoto oscillators (8) whenever the graph induced by $P$ has a globally reachable node.

Theorem V.1 (Frequency synchronization) Consider the non-uniform Kuramoto model (8) where the graph induced by $P$ has a globally reachable node. Assume that there exists $\gamma \in\left[0, \pi / 2-\varphi_{\max }[\right.$ such that the (non-empty) set of bounded phase differences $\bar{\Delta}(\gamma)$ is positively invariant. Then for every $\theta(0) \in \bar{\Delta}(\gamma)$,

1) the frequencies $\dot{\theta}_{i}(t)$ synchronize exponentially to some frequency $\dot{\theta}_{\infty} \in\left[\dot{\theta}_{\min }(0), \dot{\theta}_{\max }(0)\right]$; and

2) if $\varphi_{\max }=0$ and $P=P^{T}$, then $\dot{\theta}_{\infty}=\Omega:=\sum_{i} \omega_{i} / \sum_{i} D_{i}$ and the exponential synchronization rate is no worse than

$$
\lambda_{\mathrm{fe}}=-\lambda_{2}\left(L\left(P_{i j}\right)\right) \cos (\gamma) \cos (\angle(D \mathbf{1}, \mathbf{1}))^{2} / D_{\max } .
$$


In the definition of the convergence rate $\lambda_{\mathrm{fe}}$ in (19), the factor $\lambda_{2}\left(L\left(P_{i j}\right)\right)$ is the algebraic connectivity of the graph induced by $P=P^{T}$, the factor $1 / D_{\max }$ is the slowest time constant of the non-uniform Kuramoto system (8), the proportionality $\lambda_{\text {fe }} \sim \cos (\gamma)$ reflects the phase cohesiveness in $\bar{\Delta}(\gamma)$, and the proportionality $\lambda_{\mathrm{fe}} \sim \cos (\angle(D \mathbf{1}, \mathbf{1}))^{2}$ reflects the fact that the error coordinate $\dot{\theta}-\Omega \mathbf{1}$ is for nonuniform damping terms $D_{i}$ not orthogonal to the agreement vector $\Omega 1$. For non-zero phase shifts a small signal analysis of the non-uniform Kuramoto model (18) reveals that the natural frequency of each oscillator diminishes as $\omega_{i}-\sum_{j \neq i} P_{i j} \sin \left(\varphi_{i j}\right)$. In this case, and for symmetric coupling $P=P^{T}$, the synchronization frequency $\dot{\theta}_{\infty}$ in statement 1) will be smaller than $\dot{\theta}_{\infty}=\Omega$ in statement 2). When specialized to the classic Kuramoto model (2), statement 2) of Theorem V.1 reduces to [32, Theorem 3.1].

Proof of Theorem V.1: By differentiating the non-uniform Kuramoto model (8) we obtain the following dynamical system describing the evolution of the frequencies

$$
\frac{d}{d t} D_{i} \dot{\theta}_{i}=-\sum_{j=1}^{n} P_{i j} \cos \left(\theta_{i}-\theta_{j}+\varphi_{i j}\right)\left(\dot{\theta}_{i}-\dot{\theta}_{j}\right) .
$$

Given the matrix $P$, consider a directed weighted graph $\mathcal{G}$ induced by the matrix with elements $a_{i j}=$ $\left(P_{i j} / D_{i}\right) \cos \left(\theta_{i}-\theta_{j}+\varphi_{i j}\right)$. By assumption we have for every $\theta(0) \in \bar{\Delta}(\gamma)$ that $\theta(t) \in \bar{\Delta}(\gamma)$ for all $t \geq 0$. Consequently the weights $a_{i j}(t)=\left(P_{i j} / D_{i}\right) \cos \left(\theta_{i}(t)-\theta_{j}(t)+\varphi_{i j}\right)$ are non-degenerate, i.e., zero for $P_{i j}=0$ and strictly positive otherwise for all $t \geq 0$. Note also that system (20) evolves on the tangent space of $\mathbb{T}^{n}$, that is, the Euclidean space $\mathbb{R}^{n}$. Therefore, the dynamics (20) can be analyzed as a linear time-varying consensus protocol for the velocities $\dot{\theta}_{i}$ with state-dependent Laplacian matrix $L\left(a_{i j}\right)$ :

$$
\frac{d}{d t} \dot{\theta}=-L\left(a_{i j}\right) \dot{\theta}
$$

We analyze $L\left(a_{i j}\right)$ as if it was a just time-varying Laplacian matrix $L\left(a_{i j}(t)\right)$. At each time instance the matrix $-L\left(a_{i j}(t)\right)$ is Metzler with zero row sums, and the weights $a_{i j}(t)$ are bounded continuous functions of time that induce integrated over any non-zero time interval a graph with non-degenerate weights and a globally reachable node. It follows from the contraction property [53, Theorem 1] that $\dot{\theta}_{i}(t) \in\left[\dot{\theta}_{\min }(0), \dot{\theta}_{\max }(0)\right]$ for all $t \geq 0$ and $\theta_{i}(t)$ converge exponentially to $\dot{\theta}_{\infty}$. This proves statement 1$){ }^{1}$

In the case of zero shifts and symmetric coupling $P=P^{T}$ the frequency dynamics (21) can be reformulated as a symmetric time-varying consensus protocol with multiple rates $D$ as

$$
\frac{d}{d t} D \dot{\theta}=-L\left(w_{i j}(t)\right) \dot{\theta}
$$

where $L\left(w_{i j}(t)\right)$ is a symmetric time-varying Laplacian corresponding to a connected graph with strictly positive weights $w_{i j}(t)=P_{i j} \cos \left(\theta_{i}-\theta_{j}\right)$. It follows from statement 1$)$ that the oscillators synchronize exponentially to some frequency $\dot{\theta}_{\infty}$. Since $L\left(w_{i j}\right)$ is symmetric, it holds that $\mathbf{1}_{n}^{T} \frac{d}{d t} D \dot{\theta}=0$, or equivalently,

\footnotetext{
${ }^{1}$ We remark that in the case of smoothly time-varying natural frequencies $\omega_{i}(t)$ an additional term $\dot{\omega}(t)$ appears on the righthand side of the frequency consensus dynamics (21). If the natural frequencies are non-identical or not exponentially convergent to identical values, the oscillators clearly cannot achieve frequency synchronization and the proof of Theorem V.1 fails.
} 


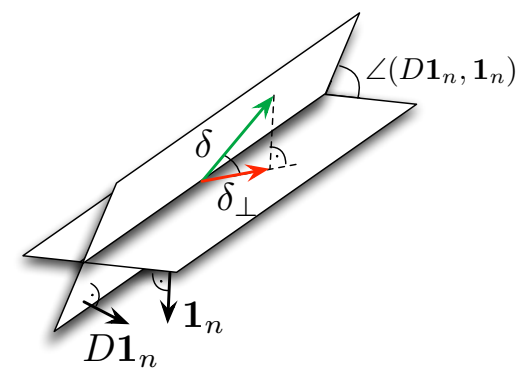

Fig. 2. Illustration of the disagreement eigenspace and the orthogonal complement of $\mathbf{1}_{n}$

$\sum_{i} D_{i} \ddot{\theta}_{i}(t)$ is a constant conserved quantity. If we apply this argument again at $\dot{\theta}_{\infty}:=\lim _{t \rightarrow \infty} \dot{\theta}_{i}(t)$, then we have $\sum_{i} D_{i} \dot{\theta}_{i}(t)=\sum_{i} D_{i} \dot{\theta}_{\infty}$, or equivalently, the frequencies synchronize exponentially to $\dot{\theta}_{\infty}=\Omega$.

In order to derive an explicit synchronization rate, consider the weighted disagreement vector $\delta=$ $\dot{\theta}-\Omega \mathbf{1}_{n}$, as an error coordinate satisfying $\mathbf{1}_{n}^{T} D \delta=\mathbf{1}_{n}^{T} D \dot{\theta}-\mathbf{1}_{n}^{T} D \Omega \mathbf{1}_{n}=0$, that is, $\delta$ lives in the weighted disagreement eigenspace of co-dimension $1_{n}$ and with normal vector $D \mathbf{1}_{n}$. Since $\Omega$ is constant and $\operatorname{ker}\left(L\left(w_{i j}\right)\right)=\operatorname{span}\left(\mathbf{1}_{n}\right)$, the weighted disagreement dynamics are obtained from (44) in $\delta$-coordinates as

$$
\frac{d}{d t} D \delta=-L\left(w_{i j}(t)\right) \delta
$$

Consider the weighted disagreement function $\delta \mapsto \delta^{T} D \delta$ and its derivative along the dynamics (23) given by

$$
\frac{d}{d t} \delta^{T} D \delta=-2 \delta^{T} L\left(w_{i j}(t)\right) \delta
$$

Since $\delta^{T} D \mathbf{1}_{n}=0$, it follows that $\delta \notin \operatorname{span}\left(\mathbf{1}_{n}\right)$ and $\delta$ can be uniquely decomposed into orthogonal components as $\delta=\left(\mathbf{1}_{n}^{T} \delta / n\right) \mathbf{1}_{n}+\delta_{\perp}$, where $\delta_{\perp}$ is the orthogonal projection of $\delta$ on the subspace orthogonal to $\mathbf{1}_{n}$. By the Courant-Fischer Theorem [54], the time derivative of the weighted disagreement function can be upper-bounded (point-wise in time) with the algebraic connectivity $\lambda_{2}\left(L\left(P_{i j}\right)\right)$ as follows:

$$
\begin{aligned}
& \frac{d}{d t} \delta^{T} D \delta=-2 \delta_{\perp}^{T} L\left(w_{i j}(t)\right) \delta_{\perp} \\
& \leq=-\left(H \delta_{\perp}\right)^{T} \cdot \operatorname{diag}\left(P_{i j} \cos \left(\theta_{i}-\theta_{j}\right)\right) \cdot\left(H \delta_{\perp}\right) \\
& \leq-\min _{\{i, j\} \in \mathcal{E}}\left\{\cos \left(\theta_{i}-\theta_{j}\right): \theta \in \bar{\Delta}(\gamma)\right\} \cdot\left(H \delta_{\perp}\right)^{T} \cdot \operatorname{diag}\left(P_{i j}\right) \cdot\left(H \delta_{\perp}\right) \leq-\lambda_{2}\left(L\left(P_{i j}\right)\right) \cos (\gamma) \cdot\left\|\delta_{\perp}\right\|_{2}^{2} .
\end{aligned}
$$

In the sequel, $\left\|\delta_{\perp}\right\|$ will be bounded by $\|\delta\|$. In order to do so, let $\mathbf{1}_{\perp}=\left(1 /\left\|\delta_{\perp}\right\|\right) \delta_{\perp}$ be the unit vector that $\delta$ is projected on (in the subspace orthogonal to $\mathbf{1}_{n}$ ). The norm of $\delta_{\perp}$ can be obtained as $\left\|\delta_{\perp}\right\|=\left\|\delta^{T} \mathbf{1}_{\perp}\right\|=\|\delta\| \cos \left(\angle\left(\delta, \mathbf{1}_{\perp}\right)\right)$. The vectors $\delta$ and $\mathbf{1}_{\perp}$ each live on $(n-1)$-dimensional linear hyperplanes with normal vectors $D \mathbf{1}_{n}$ and $\mathbf{1}_{n}$, respectively, see Figure 2 for an illustration. The angle $\angle\left(\delta, \mathbf{1}_{\perp}\right)$ is upper-bounded by $\max _{\delta} \angle\left(\delta, \mathbf{1}_{\perp}\right)$, which is said to be the dihedral angle and its sine is the gap between the two subspaces [54]. Since both hyperplanes are of co-dimension 1, we obtain the dihedral angle as the angle between the normal vectors $D \mathbf{1}_{n}$ and $\mathbf{1}_{n}$, and it follows that $\angle\left(\delta, \mathbf{1}_{\perp}\right) \leq \angle\left(D \mathbf{1}_{n}, \mathbf{1}_{n}\right)$ (with equality for $n=2$ ). In summary, we have $\|\delta\| \geq\left\|\delta_{\perp}\right\| \geq\|\delta\| \cos \left(\angle\left(D \mathbf{1}_{n}, \mathbf{1}_{n}\right)\right.$ ). 
Finally, given $D_{\min }\|\delta\|^{2} \leq \delta^{T} D \delta \leq D_{\max }\|\delta\|^{2}$ and $\lambda_{\text {fe }}$ as stated in equation (19), we obtain for the derivative of the disagreement function $\frac{d}{d t} \delta^{T} D \delta \leq-2 \lambda_{\mathrm{fe}} \delta^{T} D \delta$. An application of the Bellman-Gronwall Lemma yields $\delta(t)^{T} D \delta(t) \leq \delta(0)^{T} D \delta(0) e^{-2 \lambda_{\mathrm{fe}}(t)}$ for all $t \geq 0$. After reusing the bounds on $\delta^{T} D \delta$, we obtain that the disagreement vector $\delta(t)$ satisfies $\|\delta(t)\| \leq \sqrt{D_{\max } / D_{\min }}\|\delta(0)\| e^{-\lambda_{\text {fe }}(t)}$ for all $t \geq 0$.

\section{B. Phase Cohesiveness}

The key assumption in Theorem V.1 is that the angular distances are bounded in the set $\Delta\left(\pi / 2-\varphi_{\max }\right)$. This subsection provides two different approaches to deriving conditions for this phase cohesiveness assumption - the contraction property and ultimate boundedness arguments. The dynamical system describing the evolution of the phase differences for the non-uniform Kuramoto model (8) reads as

$$
\dot{\theta}_{i}-\dot{\theta}_{j}=\frac{\omega_{i}}{D_{i}}-\frac{\omega_{j}}{D_{j}}-\sum_{k=1}^{n}\left(\frac{P_{i k}}{D_{i}} \sin \left(\theta_{i}-\theta_{k}+\varphi_{i k}\right)-\frac{P_{j k}}{D_{j}} \sin \left(\theta_{j}-\theta_{k}+\varphi_{j k}\right)\right), i, j \in\{1, \ldots, n\} .
$$

Note that equation (24) cannot have a fixed point of the form $\dot{\theta}_{i}=\dot{\theta}_{j}$ if the following condition is not met.

Lemma V.2 (Necessary Condition on Synchronization) Consider the non-uniform Kuramoto model (8). For any two distinct $i, j \in\{1, \ldots, n\}$ there exists no solution of the form $\dot{\theta}_{i}(t)=\dot{\theta}_{j}(t), t \geq 0$, if

$$
\left|\frac{\omega_{i}}{D_{i}}-\frac{\omega_{j}}{D_{j}}\right|>\sum_{k=1}^{n}\left(\frac{P_{i k}}{D_{i}}+\frac{P_{j k}}{D_{j}}\right) .
$$

Condition (25) can be interpreted as "the coupling between oscillators $i$ and $j$ needs to dominate their non-uniformity" such that they can synchronize. For the classic Kuramoto model (2) condition (25) reduces to $K<n /(2(n-1)) \cdot\left(\omega_{i}-\omega_{j}\right)$, a necessary condition derived also in [32], [33], [25]. We remark that condition (25) is only a loose bound for synchronization since it does take into account the effect of lossy coupling induced by the phase shift $\varphi_{i j}$, which becomes obvious when expanding the sinusoidal coupling terms in (24) as in equation (18). Nevertheless, condition (25) indicates that the coupling needs to dominate the non-uniformity and possibly also disadvantageous effects of the lossy coupling.

In order to show the phase cohesiveness $\theta(t) \in \Delta\left(\pi / 2-\varphi_{\max }\right)$, the Kuramoto literature provides various methods such as quadratic Lyapunov functions [32], contraction mapping [33], geometric [24], or Hamiltonian arguments [27], [25]. Due to the non-symmetric coupling via the weights $P_{i j} / D_{i}$ and the phase shifts $\varphi_{i j}$ none of the mentioned methods appears to be easily applicable to the non-uniform Kuramoto model non-uniform Kuramoto model (8). A different approach from the literature on consensus protocols [18], [19], [39] is based on convexity and contraction and aims to show that the arc in which all phases are contained is of non-increasing length. A modification of this approach turns out to be applicable to non-uniform Kuramoto oscillators with a complete coupling graph and results in the following theorem.

Theorem V.3 (Synchronization condition I) Consider the non-uniform Kuramoto-model (8), where the graph induced by $P=P^{T}$ is complete. Assume that the minimal lossless coupling of any oscillator to 
the network is larger than a critical value, i.e.,

$\Gamma_{\min }:=n \min _{i \neq j}\left\{\frac{P_{i j}}{D_{i}} \cos \left(\varphi_{i j}\right)\right\}>\Gamma_{\text {critical }}:=\frac{1}{\cos \left(\varphi_{\max }\right)}\left(\max _{i \neq j}\left|\frac{\omega_{i}}{D_{i}}-\frac{\omega_{j}}{D_{j}}\right|+2 \max _{i \in\{1, \ldots, n\}} \sum_{j=1}^{n} \frac{P_{i j}}{D_{i}} \sin \left(\varphi_{i j}\right)\right)$.

Accordingly, define $\gamma_{\min } \in\left[0, \pi / 2-\varphi_{\max }\left[\right.\right.$ and $\left.\left.\gamma_{\max } \in\right] \pi / 2, \pi\right]$ as unique solutions to the equations $\sin \left(\gamma_{\min }\right)=\sin \left(\gamma_{\max }\right)=\cos \left(\varphi_{\max }\right) \Gamma_{\text {critical }} / \Gamma_{\min }$. Then

1) phase cohesiveness: the set $\bar{\Delta}(\gamma)$ is positively invariant for every $\gamma \in\left[\gamma_{\min }, \gamma_{\max }\right]$, and each trajectory starting in $\Delta\left(\gamma_{\max }\right)$ reaches $\bar{\Delta}\left(\gamma_{\min }\right)$; and

2) frequency synchronization: for every $\theta(0) \in \Delta\left(\gamma_{\max }\right)$, the frequencies $\dot{\theta}_{i}(t)$ synchronize exponentially to some frequency $\dot{\theta}_{\infty} \in\left[\dot{\theta}_{\min }(0), \dot{\theta}_{\max }(0)\right]$.

Condition (26) is interpreted in Remark III.3. In essence, Theorem V.3 is based on the contraction property: the positive invariance of $\bar{\Delta}(\gamma)$ is equivalent to showing that all angles $\theta_{i}(t)$ are contained in a rotating arc of non-increasing maximal length $\gamma$. This contraction analysis is similar to that of the consensus algorithms in [18], [19], [39], which derive their results on $\mathbb{R}^{n}$. Throughout the proof of Theorem V.3 we comment on different possible branches leading to slightly different conditions than (26). These branches are explored in detail in the Appendix VIII.

Remark V.4 (Reduction of Theorem V.3 to classic Kuramoto oscillators:) For the classic Kuramoto oscillators (2) the sufficient condition (26) of Theorem V.3 specializes to

$$
K>K_{\text {critical }}:=\omega_{\max }-\omega_{\min } .
$$

In other words, if $K>K_{\text {critical }}$, then for every $\theta(0) \in \Delta\left(\gamma_{\max }\right)$ the oscillators synchronize and are ultimately phase cohesive in $\bar{\Delta}\left(\gamma_{\min }\right)$, where $\left.\left.\gamma_{\max } \in\right] \pi / 2, \pi\right]$ and $\gamma_{\min } \in[0, \pi / 2[$ are the unique solutions to the equations $\sin \left(\gamma_{\min }\right)=\sin \left(\gamma_{\max }\right)=K_{\text {critical }} / K$. To the best of our knowledge, condition (27) on the coupling gain $K$ is the tightest explicit sufficient synchronization condition that has been presented in the Kuramoto literature so far. In fact, the bound (27) is close to the necessary condition for synchronization $K>K_{\text {critical }} n /(2(n-1))$ derived in Lemma V.2 and [32], [33], [25]. Obviously, for $n=2$ condition (27) is necessary and sufficient for the onset of synchronization. Other sufficient bounds given in the literature scale asymptotically with $n$, e.g., [33, Theorem 2] or [32, proof of Theorem 4.1]. To compare our condition (27) with the bounds in [32], [24], [39], we note from the proof of Theorem V.3 that our condition can be equivalently stated as follows. The set $\bar{\Delta}(\pi / 2-\gamma)$, for $\gamma \in] 0, \pi / 2]$, is positively invariant if

$$
K \geq K(\gamma):=\frac{K_{\text {critical }}}{\cos (\gamma)}=\frac{\omega_{\max }-\omega_{\text {min }}}{\cos (\gamma)} .
$$

Our bound (28) improves the bound $K>K(\gamma) n / 2$ derived in [32, proof of Theorem 4.1] via a quadratic Lyapunov function, the bound $K>K(\gamma) n /(n-2)$ derived in [39] via contraction arguments similar to ours, and the bound derived geometrically in [24, proof of Proposition 1] that, after some manipulations, can be written in our notation as $K \geq K(\gamma) \cos ((\pi / 2-\gamma) / 2) / \cos (\pi / 2-\gamma)$. Our ongoing research also 
reveals that the bound (27) is tight for a bimodal distribution of the natural frequencies $\omega_{i} \in\left\{\omega_{\max }, \omega_{\min }\right\}$ and also satisfies the implicit consistency conditions in [29]. Thus, (27) is a necessary and sufficient condition for synchronization when the natural frequencies $\omega_{i}$ are only known to be contained in $\left[\omega_{\min }, \omega_{\max }\right]$. We elaborate on this interesting circle of ideas in a separate publication [55].

In summary, condition (26) in Theorem V.3 improves the known [32], [33], [25], [24], [39] sufficient conditions for synchronization of classic Kuramoto oscillators, and it is a necessary and sufficient if the particular distribution of the natural frequencies $\omega_{i} \in\left[\omega_{\min }, \omega_{\max }\right]$ is unknown.

Proof of Theorem V.3: We start by proving the positive invariance of $\bar{\Delta}(\gamma)$ for $\gamma \in[0, \pi]$. Recall the geodesic distance between two angles on $\mathbb{T}^{1}$ and define the non-smooth function $V: \mathbb{T}^{n} \rightarrow[0, \pi]$ by

$$
V(\psi)=\max \left\{\left|\psi_{i}-\psi_{j}\right| \mid i, j \in\{1, \ldots, n\}\right\} .
$$

By assumption, the angles $\theta_{i}(t)$ belong to the set $\bar{\Delta}(\gamma)$ at time $t=0$, that is, they are all contained in an arc of length $\gamma \in[0, \pi]$. In this case, $V(\psi)$ can equivalently be written as maximum over a set of differentiable functions, that is, $V(\psi)=\max \left\{\psi_{i}-\psi_{j} \mid i, j \in\{1, \ldots, n\}\right\}$. The arc containing all angles has two boundary points: a counterclockwise maximum and a counterclockwise minimum. If we let $I_{\max }(\psi)$ (respectively $I_{\min }(\psi)$ ) denote the set indices of the angles $\psi_{1}, \ldots, \psi_{n}$ that are equal to the counterclockwise maximum (respectively the counterclockwise minimum), then we may write

$$
V(\psi)=\psi_{m^{\prime}}-\psi_{\ell^{\prime}}, \quad \text { for all } m^{\prime} \in I_{\max }(\psi) \text { and } \ell^{\prime} \in I_{\min }(\psi) \text {. }
$$

We aim to show that all angles remain in $\bar{\Delta}(\gamma)$ for all subsequent times $t>0$. Note that $\theta(t) \in \bar{\Delta}(\gamma)$ if and only if $V(\theta(t)) \leq \gamma$. Therefore, $\bar{\Delta}(\gamma)$ is positively invariant if and only if $V(\theta(t))$ does not increase at any time $t$ such that $V(\theta(t))=\gamma$. The upper Dini derivative of $V(\theta(t))$ along the dynamical system (24) is given as in [19, Lemma 2.2]

$$
D^{+} V(\theta(t))=\lim _{h \downarrow 0} \sup \frac{V(\theta(t+h))-V(\theta(t))}{h}=\dot{\theta}_{m}(t)-\dot{\theta}_{\ell}(t),
$$

where $m \in I_{\max }(\theta(t))$ and $\ell \in I_{\min }(\theta(t))$ are indices with the properties that

$$
\dot{\theta}_{m}(t)=\max \left\{\dot{\theta}_{m^{\prime}}(t) \mid m^{\prime} \in I_{\max }(\theta(t))\right\}, \text { and } \quad \dot{\theta}_{\ell}(t)=\min \left\{\dot{\theta}_{\ell^{\prime}}(t) \mid \ell^{\prime} \in I_{\min }(\theta(t))\right\} .
$$

Written out in components (in the expanded form (18)) $D^{+} V(\theta(t))$ takes the form

$$
\begin{aligned}
D^{+} V(\theta(t))= & \frac{\omega_{m}}{D_{m}}-\frac{\omega_{\ell}}{D_{\ell}}-\sum_{k=1}^{n}\left(a_{m k} \sin \left(\theta_{m}(t)-\theta_{k}(t)\right)+a_{\ell k} \sin \left(\theta_{k}(t)-\theta_{\ell}(t)\right)\right) \\
& -\sum_{k=1}^{n}\left(b_{m k} \cos \left(\theta_{m}(t)-\theta_{k}(t)\right)-b_{\ell k} \cos \left(\theta_{\ell}(t)-\theta_{k}(t)\right)\right)
\end{aligned}
$$

where we used the abbreviations $a_{i k}:=P_{i k} \cos \left(\varphi_{i k}\right) / D_{i}$ and $b_{i k}:=P_{i k} \sin \left(\varphi_{i k}\right) / D_{i}$. The equality $V(\theta(t))=\gamma$ implies that, measuring distances counterclockwise and modulo additional terms equal to multiples of $2 \pi$, we have $\theta_{m}(t)-\theta_{\ell}(t)=\gamma, 0 \leq \theta_{m}(t)-\theta_{k}(t) \leq \gamma$, and $0 \leq \theta_{k}(t)-\theta_{\ell}(t) \leq \gamma$. To 
simplify the notation in the subsequent arguments, we do not aim at the tightest and least conservative bounding of the two sums on the right-hand side of (29) and continue as follows. ${ }^{2}$

Since both sinusoidal terms on the right-hand side of (29) are positive, they can be lower-bounded as

$$
\begin{gathered}
a_{m k} \sin \left(\theta_{m}(t)-\theta_{k}(t)\right)+a_{\ell k} \sin \left(\theta_{k}(t)-\theta_{\ell}(t)\right) \geq \min _{i \in\{m, \ell\} \backslash\{k\}}\left\{a_{i k}\right\}\left(\sin \left(\theta_{m}(t)-\theta_{k}(t)\right)+\sin \left(\theta_{k}(t)-\theta_{\ell}(t)\right)\right) \\
=2 \min _{i \in\{m, \ell\} \backslash\{k\}}\left\{a_{i k}\right\} \sin \left(\frac{\theta_{m}(t)-\theta_{\ell}(t)}{2}\right) \cos \left(\frac{\theta_{m}(t)+\theta_{\ell}(t)}{2}-\theta_{k}(t)\right) \\
\geq 2 \min _{i \in\{m, \ell\} \backslash\{k\}}\left\{a_{i k}\right\} \sin \left(\frac{\gamma}{2}\right) \cos \left(\frac{\gamma}{2}\right)=\min _{i \in\{m, \ell\} \backslash\{k\}}\left\{a_{i k}\right\} \sin (\gamma),
\end{gathered}
$$

where we applied the trigonometric identities $\sin (x)+\sin (y)=2 \sin \left(\frac{x+y}{2}\right) \cos \left(\frac{x-y}{2}\right)$ and $2 \sin (x) \cos (y)=$ $\sin (x-y)+\sin (x+y)$. The cosine terms in (29) can be lower bounded in $\bar{\Delta}(\gamma)$ as $b_{m k} \cos \left(\theta_{m}(t)-\right.$ $\left.\theta_{k}(t)\right)-b_{\ell k} \cos \left(\theta_{\ell}(t)-\theta_{k}(t)\right) \geq-b_{m k}-b_{\ell k}$. In summary, $D^{+} V(\theta(t))$ in (29) can be upper bounded by

$$
\begin{aligned}
D^{+} V(\theta(t)) & \leq \frac{\omega_{m}}{D_{m}}-\frac{\omega_{\ell}}{D_{\ell}}-\sum_{k=1}^{n} \min _{i \in\{m, \ell\} \backslash\{k\}}\left\{a_{i k}\right\} \sin (\gamma)+\sum_{k=1}^{n} b_{m k}+\sum_{k=1}^{n} b_{\ell k} \\
& \leq \max _{i \neq j}\left|\frac{\omega_{i}}{D_{i}}-\frac{\omega_{j}}{D_{j}}\right|-n \min _{i \neq j}\left\{\frac{P_{i j}}{D_{i}} \cos \left(\varphi_{i j}\right)\right\} \sin (\gamma)+2 \max _{i \in\{1, \ldots, n\}} \sum_{j=1}^{n} b_{i j},
\end{aligned}
$$

where we further maximized the coupling terms and the differences in natural frequencies over all possible pairs $\{m, \ell\}$. It follows that $V(\theta(t))$ is non-increasing for all $\theta(t) \in \bar{\Delta}(\gamma)$ if

$$
\Gamma_{\min } \sin (\gamma) \geq \cos \left(\varphi_{\max }\right) \Gamma_{\text {critical }},
$$

where $\Gamma_{\min }$ and $\Gamma_{\text {critical }}$ are defined in (26). The left-hand side of (30) is a strictly concave function of $\gamma \in$ $[0, \pi]$. Thus, there exists an open set of arc lengths $\gamma$ including $\gamma^{*}=\pi / 2-\varphi_{\max }$ satisfying inequality (30) if and only if inequality (30) is true at $\gamma^{*}=\pi / 2-\varphi_{\max }$ with the strict inequality sign, which corresponds to condition (26) in the statement of Theorem V.3. Additionally, if these two equivalent statements are true, then $V(\theta(t))$ is non-increasing in $\bar{\Delta}(\gamma)$ for all $\gamma \in\left[\gamma_{\min }, \gamma_{\max }\right]$, where $\gamma_{\min } \in\left[0, \pi / 2-\varphi_{\max }[\right.$ and $\left.\left.\gamma_{\max } \in\right] \pi / 2, \pi\right]$ are given as unique solutions to inequality (30) with equality sign. Moreover, $V(\theta(t))$ is strictly decreasing in $\bar{\Delta}(\gamma)$ for all $\gamma \in] \gamma_{\min }, \gamma_{\max }[$. This concludes the proof of statement 1) and ensures that for every $\theta(0) \in \Delta\left(\gamma_{\max }\right)$, there exists $T \geq 0$ such that $\theta(t) \in \bar{\Delta}\left(\pi / 2-\varphi_{\max }\right)$ for all $t \geq T$. Thus, the positive invariance assumption of Theorem V.1 is satisfied, and statement 2) of Theorem V.3 follows.

In summary, Theorem V.3 presents sufficient conditions for the synchronization of the non-uniform Kuramoto model and is based on the bound (26). Condition (26) is a worst-case bound, both on the parameters and on the initial angles. In the remainder of this section, we aim at deriving a two-norm type bound and require only connectivity of the graph induced by $P=P^{T}$ and not necessarily completeness.

\footnotetext{
${ }^{2}$ Besides tighter bounding of the right-hand side of (29), the proof can alternatively be continued by adding and subtracting the coupling with zero phase shifts in (29) or by noting that the right-hand side of (29) is a convex function of $\theta_{k} \in\left[\theta_{\ell}, \theta_{m}\right]$ that achieves its maximum at the boundary $\theta_{k} \in\left\{\theta_{\ell}, \theta_{m}\right\}$. If the analysis is restricted to $\gamma \in[0, \pi / 2]$, the term $b_{m k}$ can be dropped.
} 
We start our discussion with some preliminary notation and concepts. The following analysis is formally carried out for the complete graph, but, without loss of generality, we assume that some weights $P_{i j}=P_{j i}$ can be zero and the non-zero weights $P=P^{T}$ induce a connected graph. Let $H \in \mathbb{R}^{n(n-1) / 2 \times n}$ be the incidence matrix of the complete graph with $n$ nodes and recall that for a vector $x \in \mathbb{R}^{n}$ the vector of all difference variables is $H x=\left(x_{2}-x_{1}, \ldots\right)$. The phase difference dynamics (24) (with the sinusoidal coupling expanded as in (18)) can be reformulated in a compact vector notation as

$$
\frac{d}{d t} H \theta=H D^{-1} \omega-H D^{-1} H^{T} \operatorname{diag}\left(P_{i j} \cos \left(\varphi_{i j}\right)\right) \sin (H \theta)-H X,
$$

where $X \in \mathbb{R}^{n}$ is the vector of lossy coupling with components $X_{i}=\sum_{j=1}^{n}\left(P_{i j} / D_{i}\right) \sin \left(\varphi_{i j}\right) \cos \left(\theta_{i}-\theta_{j}\right)$ and $\sin (H \theta)$ is the multivariable sine. The set of differential equations (31) is well defined on $\mathbb{T}^{n}$ : the left-hand side of (31) is the vector of frequency differences $H \dot{\theta}=\left(\dot{\theta}_{2}-\dot{\theta}_{1}, \ldots\right)$ taking values in the tangent space to $\mathbb{T}^{n}$, and the right-hand side of (31) is a well-posed vector-valued function of $\theta \in \mathbb{T}^{n}$.

With slight abuse of notation, we denote the two-norm of the vector of all geodesic distances by $\|H \theta\|_{2}=\left(\sum_{i} \sum_{j}\left|\theta_{i}-\theta_{j}\right|^{2}\right)^{1 / 2}$, and aim at ultimately bounding the evolution of $\|H \theta(t)\|_{2}$. Following a classic Kuramoto analysis [33], [28], [27], [25], we note that the non-uniform Kuramoto model (8) with $\omega \equiv \mathbf{0}$ constitutes a Hamiltonian system with the Hamiltonian $\left.U(\theta)\right|_{\omega=0}$ defined in equation (6). An analysis of (31) by Hamiltonian arguments is possible, but results in very conservative conditions. In the recent Kuramoto literature [32], [33], a different Lyapunov function considered for the uniform Kuramoto model (2) evolving on $\mathbb{R}^{n}$ is simply $\|H \theta\|_{2}^{2}$. Unfortunately, in the case of non-uniform rates $D_{i}$ this function's Lie derivative is sign-indefinite. However, it is possible to identify a similar Lyapunov function that has a Lie derivative with symmetric coupling. Consider the function $\mathcal{W}: \mathbb{T}^{n} \rightarrow \mathbb{R}$ defined by

$$
\mathcal{W}(\theta)=\frac{1}{4} \sum_{i=1}^{n} \sum_{j=1}^{n} D_{i} D_{j}\left|\theta_{i}-\theta_{j}\right|^{2} .
$$

A Lyapunov analysis of system (31) via the Lyapunov function $\mathcal{W}$ leads to the following theorem.

Theorem V.5 (Synchronization condition II) Consider the non-uniform Kuramoto model (8), where the graph induced by $P=P^{T}$ is connected. Let $H \in \mathbb{R}^{n(n-1) / 2 \times n}$ be the incidence matrix of the complete graph and assume that the algebraic connectivity of the lossless coupling is larger than a critical value, i.e., $\lambda_{2}\left(L\left(P_{i j} \cos \left(\varphi_{i j}\right)\right)\right)>\lambda_{\text {critical }}:=\frac{\left\|H D^{-1} \omega\right\|_{2}+\sqrt{n}\left\|\left[\sum_{j=1}^{n} \frac{P_{1 j}}{D_{1}} \sin \left(\varphi_{1 j}\right), \ldots, \sum_{j=1}^{n} \frac{P_{n j}}{D_{n}} \sin \left(\varphi_{n j}\right)\right]\right\|_{2}}{\cos \left(\varphi_{\max }\right)(\kappa / n) \alpha / \max _{i \neq j}\left\{D_{i} D_{j}\right\}}$,

where $\kappa:=\sum_{k=1}^{n} D_{k}$ and $\alpha:=\sqrt{\min _{i \neq j}\left\{D_{i} D_{j}\right\} / \max _{i \neq j}\left\{D_{i} D_{j}\right\}}$.

Accordingly, define $\left.\left.\gamma_{\max } \in\right] \pi / 2-\varphi_{\max }, \pi\right]$ and $\gamma_{\min } \in\left[0, \pi / 2-\varphi_{\max }[\right.$ to be the unique solutions to the equations $\operatorname{sinc}\left(\gamma_{\max }\right) / \operatorname{sinc}\left(\pi / 2-\varphi_{\max }\right)=\sin \left(\gamma_{\min }\right) / \cos \left(\varphi_{\max }\right)=\lambda_{\text {critical }} / \lambda_{2}\left(L\left(P_{i j} \cos \left(\varphi_{i j}\right)\right)\right)$. Then,

1) phase cohesiveness: the set $\left\{\theta \in \Delta(\pi):\|H \theta\|_{2} \leq \gamma\right\}$ is positively invariant for every $\gamma \in$ $\left[\gamma_{\min }, \alpha \gamma_{\max }\right]$, and each trajectory starting in $\left\{\theta \in \Delta(\pi):\|H \theta(0)\|_{2}<\alpha \gamma_{\max }\right\}$ reaches $\{\theta \in$ $\left.\Delta(\pi):\|H \theta\|_{2} \leq \gamma_{\min }\right\} ;$ and 
2) frequency synchronization: for every $\theta(0) \in \Delta(\pi)$ with $\|H \theta(0)\|_{2}<\alpha \gamma_{\max }$ the frequencies $\dot{\theta}_{i}(t)$ synchronize exponentially to some frequency $\dot{\theta}_{\infty} \in\left[\dot{\theta}_{\min }(0), \dot{\theta}_{\max }(0)\right]$. Moreover, if $\varphi_{\max }=0$, then $\dot{\theta}_{\infty}=\Omega$ and the exponential synchronization rate is no worse than $\lambda_{\mathrm{fe}}$ as defined in equation (19).

Remark V.6 (Physical interpretation of Theorem V.5:) In condition (33), $\left\|\left[\ldots, \sum_{j=1}^{n} \frac{P_{i j}}{D_{i}} \sin \left(\varphi_{i j}\right), \ldots\right]\right\|_{2}$ is the two-norm of the vector containing the lossy coupling, $\left\|H D^{-1} \omega\right\|_{2}=\left\|\left(\omega_{2} / D_{2}-\omega_{1} / D_{1}, \ldots\right)\right\|_{2}$ corresponds to the non-uniformity in the natural frequencies, $\lambda_{2}\left(L\left(P_{i j} \cos \left(\varphi_{i j}\right)\right)\right)$ is the algebraic connectivity induced by the lossless coupling, $\cos \left(\varphi_{\max }\right)=\sin \left(\pi / 2-\varphi_{\max }\right)$ reflects again the phase cohsiveness in $\Delta\left(\pi / 2-\varphi_{\max }\right)$, and $(\kappa / n) \alpha / \max _{i \neq j}\left\{D_{i} D_{j}\right\}$ weights the non-uniformity in the time constants $D_{i}$. The gap in condition (33) yields a again practical stability result determining the initial and ultimate phase cohesiveness. Condition (33) can be extended to non-reduced power network models [42].

Remark V.7 (Reduction of Theorem V.5 to classic Kuramoto oscillators:) For classic Kuramoto oscillators (2), condition (33) reduces to $K>K_{\text {critical }}^{*}:=H \omega \|_{2}$, which is a more conservative bound than $K>K_{\text {critical }}=\omega_{\max }-\omega_{\min }$ presented in (27). It follows that the oscillators synchronize for $\|H \theta(0)\|_{2}<$ $\gamma_{\max }$ and are ultimately phase cohesive in $\|H \theta\|_{2} \leq \gamma_{\min }$, where $\left.\left.\gamma_{\max } \in\right] \pi / 2, \pi\right]$ and $\gamma_{\min } \in[0, \pi / 2[$ are the unique solutions to $(\pi / 2) \operatorname{sinc}\left(\gamma_{\max }\right)=\sin \left(\gamma_{\min }\right)=K_{\text {critical }}^{*} / K$. The Lyapunov function $\mathcal{W}(\theta)$ reduces to the one used in [32], [33] and can also be used to prove [32, Theorem 4.2] and [33, Theorem 1].

Recall from Section I that angular differences are well defined for $\theta \in \Delta(\pi)$. Hence, for $\theta \in \Delta(\pi)$, the vector of phase differences is $H \theta=\left(\theta_{2}-\theta_{1}, \ldots\right) \in \mathbb{R}^{n(n-1) / 2}$, and the function $\mathcal{W}$ defined in (32) can be rewritten as the function $H \theta \mapsto W(H \theta)$ defined by

$$
\mathcal{W}(\theta)=\frac{1}{4} \sum_{i=1}^{n} \sum_{j=1}^{n} D_{i} D_{j}\left|\theta_{i}-\theta_{j}\right|^{2}=\frac{1}{2}(H \theta)^{T} \operatorname{diag}\left(D_{i} D_{j}\right)(H \theta)=: W(H \theta) .
$$

The derivative of $W(H \theta)$ along trajectories of system (31) is then given by

$$
\begin{aligned}
\dot{W}(H \theta)=(H \theta)^{T} \operatorname{diag}\left(D_{i} D_{j}\right) H D^{-1} \omega-(H \theta)^{T} \operatorname{diag}\left(D_{i} D_{j}\right) H X & \\
& -(H \theta)^{T} \operatorname{diag}\left(D_{i} D_{j}\right) H D^{-1} H^{T} \operatorname{diag}\left(P_{i j} \cos \left(\varphi_{i j}\right)\right) \sin (H \theta) .
\end{aligned}
$$

A component-wise analysis of the last term on the right-hand side of (35) yields a "diagonal" simplification.

Lemma V.8 Let $P=P^{T} \in \mathbb{R}^{n \times n}, \theta \in \Delta(\pi)$, and $\kappa:=\sum_{k=1}^{n} D_{k}$. Then it holds that

$$
(H \theta)^{T} \operatorname{diag}\left(D_{i} D_{j}\right) H D^{-1} H^{T} \operatorname{diag}\left(P_{i j} \cos \left(\varphi_{i j}\right)\right) \sin (H \theta)=\kappa(H \theta)^{T} \operatorname{diag}\left(P_{i j} \cos \left(\varphi_{i j}\right)\right) \sin (H \theta) .
$$

Proof: The left-hand side of equation (36) reads component-wise as

$\sum_{i} \sum_{j} \sum_{k}\left(\theta_{i}-\theta_{j}\right)\left(P_{i k} \cos \left(\varphi_{i k}\right) D_{j}\right) \sin \left(\theta_{i}-\theta_{k}\right)+\sum_{i} \sum_{j} \sum_{k}\left(\theta_{i}-\theta_{j}\right)\left(P_{j k} \cos \left(\varphi_{j k}\right) D_{i}\right) \sin \left(\theta_{k}-\theta_{j}\right)$, where all indices satisfy $i, j, k \in\{1, \ldots, n\}$. An manipulation of the indices in both sums yields $\sum_{i} \sum_{k} \sum_{j}\left(\theta_{i}-\theta_{k}\right)\left(P_{i j} \cos \left(\varphi_{i j}\right) D_{k}\right) \sin \left(\theta_{i}-\theta_{j}\right)+\sum_{k} \sum_{j} \sum_{i}\left(\theta_{k}-\theta_{j}\right)\left(P_{i j} \cos \left(\varphi_{i j}\right) D_{k}\right) \sin \left(\theta_{i}-\theta_{j}\right)$. 
Finally, the two sums can be added and simplify to $\sum_{i} \sum_{k} \sum_{j}\left(P_{i j} \cos \left(\varphi_{i j}\right) D_{k}\right)\left(\theta_{i}-\theta_{j}\right) \sin \left(\theta_{i}-\theta_{j}\right)$, which equals the right-hand side of equation (36) written in components.

The following lemma will help us to upper-bound the derivative $\dot{W}(H \theta)$ by the algebraic connectivity.

Lemma V.9 Consider a connected graph with $n$ nodes induced by $A=A^{T} \in \mathbb{R}^{n \times n}$ with incidence matrix $B$ and Laplacian $L\left(A_{i j}\right)$. For any $x \in \mathbb{R}^{n}$, it holds that $(B x)^{T} \operatorname{diag}\left(A_{i j}\right)(B x) \geq\left(\lambda_{2}\left(L\left(A_{i j}\right)\right) / n\right)\|B x\|_{2}^{2}$.

Proof: Let $H$ be the incidence matrix of the complete graph. The Laplacian of the complete graph with uniform weights is then given by $\left(n \cdot I_{n}-\mathbf{1}_{n} \mathbf{1}_{n}^{T}\right)=H^{T} H$, and the projection of $x \in \mathbb{R}^{n}$ on the subspace orthogonal to $\mathbf{1}_{n}$ is $x_{\perp}=\left(I_{n}-(1 / n) \mathbf{1}_{n} \mathbf{1}_{n}^{T}\right) x=(1 / n) H^{T} H x$. Consider now the inequality

$$
\begin{aligned}
& (B x)^{T} \operatorname{diag}\left(A_{i j}\right)(B x)=x^{T} B^{T} \operatorname{diag}\left(A_{i j}\right) B x=x^{T} L\left(A_{i j}\right) x \\
& \geq \lambda_{2}\left(L\left(A_{i j}\right)\right)\left\|x_{\perp}\right\|_{2}^{2}=\frac{\lambda_{2}\left(L\left(A_{i j}\right)\right)}{n^{2}}\left\|H^{T} H x\right\|_{2}^{2}=\frac{\lambda_{2}\left(L\left(A_{i j}\right)\right)}{n^{2}}(H x)^{T} H H^{T}(H x) .
\end{aligned}
$$

In order to continue, first note that $H H^{T}$ and the complete graph's Laplacian $H^{T} H$ have the same eigenvalues, namely $n$ and 0 . Second, $\operatorname{range}(H)$ and $\operatorname{ker}\left(H^{T}\right)$ are orthogonal complements. It follows that $(H x)^{T} H H^{T}(H x)=n\|H x\|_{2}^{2}$. Finally, note that $\|H x\|_{2}^{2} \geq\|B x\|_{2}^{2}$ and the lemma follows.

Given Lemma V.8 and Lemma V.9 about the time derivative of $W(H \theta)$, we are now in a position to prove Theorem V.5 via standard Lyapunov and ultimate boundedness arguments.

Proof of Theorem V.5: Assume that $\theta(0) \in \mathcal{S}(\rho):=\left\{\theta \in \Delta(\pi):\|H \theta\|_{2} \leq \rho\right\}$ for some $\left.\rho \in\right] 0, \pi[$. In the following, we will show under which conditions and for which values of $\rho$ the set $\mathcal{S}(\rho)$ is positively invariant. For $\theta \in \mathcal{S}(\rho)$ and since $\|H \theta\|_{\infty} \leq\|H \theta\|_{2}$, it follows that $\theta \in \bar{\Delta}(\rho)$ and $1 \geq \operatorname{sinc}\left(\theta_{i}-\theta_{j}\right) \geq$ $\operatorname{sinc}(\rho)$. Thus, for $\theta \in \mathcal{S}(\rho)$, the inequality $\left(\theta_{i}-\theta_{j}\right) \sin \left(\theta_{i}-\theta_{j}\right) \geq\left(\theta_{i}-\theta_{j}\right)^{2} \operatorname{sinc}(\rho)$ and Lemma V.8 yield an upper bound on the right-hand side of (35):

$\dot{W}(H \theta) \leq(H \theta)^{T} \operatorname{diag}\left(D_{i} D_{j}\right) H D^{-1} \omega-(H \theta)^{T} \operatorname{diag}\left(D_{i} D_{j}\right) H X-\kappa \operatorname{sinc}(\rho)(H \theta)^{T} \operatorname{diag}\left(P_{i j} \cos \left(\varphi_{i j}\right)\right)(H \theta)$. Note that $\|H X\|_{2}$ is lower bounded as

$$
\|H X\|_{2}=\sqrt{X^{T} H^{T} H X} \geq \sqrt{\lambda_{\max }\left(H^{T} H\right)}\|X\|_{2} \geq \sqrt{n}\left\|\left[\ldots, \sum_{j} \frac{P_{i j}}{D_{i}} \sin \left(\varphi_{i j}\right), \ldots\right]\right\|_{2}=: \tilde{X} .
$$

This lower bound $\tilde{X}$ together with Lemma V.9 leads to the following upper bound on $\dot{W}(H \theta)$ :

$$
\dot{W}(H \theta) \leq\|H \theta\|_{2} \max _{i \neq j}\left\{D_{i} D_{j}\right\}\left(\left\|H D^{-1} \omega\right\|_{2}+\tilde{X}\right)-(\kappa / n) \operatorname{sinc}(\rho) \lambda_{2}\left(L\left(P_{i j} \cos \left(\varphi_{i j}\right)\right)\|H \theta\|_{2}^{2} .\right.
$$

Note that the right-hand side of (37) is strictly negative for

$$
\|H \theta\|_{2}>\mu_{c}:=\frac{\max _{i \neq j}\left\{D_{i} D_{j}\right\}\left(\left\|H D^{-1} \omega\right\|_{2}+\tilde{X}\right)}{(\kappa / n) \operatorname{sinc}(\rho) \lambda_{2}\left(L\left(P_{i j} \cos \left(\varphi_{i j}\right)\right)\right)} .
$$

In the following we apply standard Lyapunov and ISS arguments. Pick $\mu \in] 0, \rho[$. If

$$
\mu>\mu_{c}=\frac{\max _{i \neq j}\left\{D_{i} D_{j}\right\}\left(\left\|H D^{-1} \omega\right\|_{2}+\tilde{X}\right)}{(\kappa / n) \operatorname{sinc}(\rho) \lambda_{2}\left(L\left(P_{i j} \cos \left(\varphi_{i j}\right)\right)\right)},
$$


then for all $\|H \theta\|_{2} \in[\mu, \rho]$, the right-hand side of (37) is upper-bounded by

$$
\dot{W}(H \theta) \leq-\left(1-\left(\mu_{c} / \mu\right)\right) \cdot(\kappa / n) \operatorname{sinc}(\rho) \lambda_{2}\left(L\left(P_{i j} \cos \left(\varphi_{i j}\right)\right)\right)\|H \theta\|_{2}^{2} .
$$

Note that $W(H \theta)$ defined in (34) can easily be upper and lower bounded by constants multiplying $\|H \theta\|_{2}^{2}$ :

$$
\min _{i \neq j}\left\{D_{i} D_{j}\right\}\|H \theta\|_{2}^{2} \leq 2 \cdot W(H \theta) \leq \max _{i \neq j}\left\{D_{i} D_{j}\right\}\|H \theta\|_{2}^{2} .
$$

To guarantee the ultimate boundedness of $H \theta$, two sublevel sets of $W(H \theta)$ have to be fitted into $\{H \theta$ : $\left.\|H \theta\|_{2} \in[\mu, \rho]\right\}$ where $\dot{W}(H \theta)$ is strictly negative. This is possible if [51, equation (4.41)]

$$
\mu<\sqrt{\min _{i \neq j}\left\{D_{i} D_{j}\right\} / \max _{i \neq j}\left\{D_{i} D_{j}\right\}} \cdot \rho=\alpha \rho .
$$

Ultimate boundedness arguments [51, Theorem 4.18] imply that, for every $\|H \theta(0)\|_{2} \leq \alpha \rho$, there is $T \geq 0$ such that $\|H \theta(t)\|_{2}$ is strictly decreasing for $t \in[0, T]$ and $\|H \theta(t)\|_{2} \leq \mu / \alpha$ for all $t \geq T$. If we choose $\mu=\alpha \gamma$ with $\left.\gamma \in] 0, \pi / 2-\varphi_{\max }\right]$, then equation (40) reduces to $\rho>\gamma$ and (38) reduces to the condition

$$
\lambda_{2}\left(L\left(P_{i j} \cos \left(\varphi_{i j}\right)\right)\right)>\frac{\max _{i \neq j}\left\{D_{i} D_{j}\right\}\left(\left\|H D^{-1} \omega\right\|_{2}+\tilde{X}\right)}{\alpha \gamma(\kappa / n) \operatorname{sinc}(\rho)}=\lambda_{\text {critical }} \frac{\cos \left(\varphi_{\max }\right)}{\gamma \operatorname{sinc}(\rho)},
$$

where $\lambda_{\text {critical }}$ is as defined in equation (33). Now, we perform a final analysis of the bound (41). The right-hand side of (41) is an increasing function of $\rho$ and decreasing function of $\gamma$ that diverges to $\infty$ as $\rho \uparrow \pi$ or $\gamma \downarrow 0$. Therefore, there exists some $(\rho, \gamma)$ in the convex set $\Lambda:=\{(\rho, \gamma): \rho \in] 0, \pi[, \gamma \in$ ] $\left.\left.0, \pi / 2-\varphi_{\max }\right], \gamma<\rho\right\}$ satisfying equation (41) if and only if equation (41) is true at $\rho=\gamma=\pi / 2-\varphi_{\max }$, where the right-hand side of (41) achieves its infimum in $\Lambda$. The latter condition is equivalent to inequality (33). Additionally, if these two equivalent statements are true, then there exists an open set of points in $\Lambda$ satisfying (41), which is bounded by the unique curve that satisfies equation (41) with the equality sign, namely $f(\rho, \gamma)=0$, where $f: \Lambda \rightarrow \mathbb{R}, f(\rho, \gamma):=\gamma \operatorname{sinc}(\rho) / \cos \left(\varphi_{\max }\right)-\lambda_{\text {critical }} / \lambda_{2}\left(L\left(P_{i j} \cos \left(\varphi_{i j}\right)\right)\right)$. Consequently, for every $(\rho, \gamma) \in\{(\rho, \gamma) \in \Lambda: f(\rho, \gamma)>0\}$, it follows for $\|H \theta(0)\|_{2} \leq \alpha \rho$ that there is $T \geq 0$ such that $\|H \theta(t)\|_{2} \leq \gamma$ for all $t \geq T$. The supremum value for $\rho$ is obviously given by $\left.\left.\rho_{\max } \in\right] \pi / 2-\varphi_{\max }, \pi\right]$ solving the equation $f\left(\rho_{\max }, \pi / 2-\varphi_{\max }\right)=0$ and the corresponding infimum of $\gamma$ by $\gamma_{\min } \in\left[0, \pi / 2-\varphi_{\max }\left[\right.\right.$ solving the equation $f\left(\gamma_{\min }, \gamma_{\min }\right)=0$.

This proves statement 1) (where we replaced $\rho_{\max }$ by $\gamma_{\max }$ ) and shows that there is $T \geq 0$ such that $\|H \theta(t)\|_{\infty} \leq\|H \theta(t)\|_{2}<\pi / 2-\varphi_{\max }$ for all $t \geq T$. Statement 2) follows then from Theorem V.1.

\section{Phase Synchronization}

For identical natural frequencies and zero phase shifts, the practical stability results in Theorem V.3 and Theorem V.5 imply $\gamma_{\min } \downarrow 0$, i.e., phase synchronization of the non-uniform Kuramoto oscillators (8).

Theorem V.10 (Phase synchronization) Consider the non-uniform Kuramoto model (8), where the graph induced by $P$ has a globally reachable node, $\varphi_{\max }=0$, and $\omega_{i} / D_{i}=\bar{\omega}$ for all $i \in\{1, \ldots, n\}$.

Then for every $\theta(0) \in \bar{\Delta}(\gamma)$ with $\gamma \in[0, \pi[$, 
1) the phases $\theta_{i}(t)$ synchronize exponentially to $\theta_{\infty}(t) \in\left[\theta_{\min }(0), \theta_{\max }(0)\right]+\bar{\omega} t$, and

2) if $P=P^{T}$, then the phases $\theta_{i}(t)$ synchronize exponentially to the weighted mean angle $\theta_{\infty}(t)=$ $\sum_{i} D_{i} \theta_{i}(0) / \sum_{i} D_{i}+\bar{\omega} t$ at a rate no worse than

$$
\lambda_{\mathrm{ps}}=-\lambda_{2}\left(L\left(P_{i j}\right)\right) \operatorname{sinc}(\gamma) \cos (\angle(D \mathbf{1}, \mathbf{1}))^{2} / D_{\max } .
$$

The worst-case phase synchronization rate $\lambda_{\mathrm{ps}}$ can be interpreted similarly as the terms in (19), where $\operatorname{sinc}(\gamma)$ corresponds to the initial phase cohesiveness in $\bar{\Delta}(\gamma)$. For classic Kuramoto oscillators (2) statements 1) and 2) can be reduced to the Kuramoto results found in [19] and Theorem 1 in [33].

Proof of Theorem V.10: First we proof statement 1). Consider again the Lyapunov function $V(\theta(t))$ from the proof of Theorem V.3. The Dini derivative in the case $\varphi_{\max }=0$ and $\omega_{i} / D_{i}=\bar{\omega}$ is simply

$$
D^{+} V(\theta(t))=-\sum_{k=1}^{n}\left(\frac{P_{m k}}{D_{m}} \sin \left(\theta_{m}(t)-\theta_{k}(t)\right)+\frac{P_{\ell k}}{D_{\ell}} \sin \left(\theta_{k}(t)-\theta_{\ell}(t)\right)\right) .
$$

Both sinusoidal terms are positive for $\theta(t) \in \bar{\Delta}(\gamma), \gamma \in[0, \pi[$. Thus, $V(\theta(t)$ is non-increasing, and $\bar{\Delta}(\gamma)$ is positively invariant. Therefore, the term $a_{i j}(t)=\left(P_{i j} / D_{i}\right) \operatorname{sinc}\left(\theta_{i}(t)-\theta_{j}(t)\right)$ is strictly positive for all $t \geq 0$, and after changing to a rotating frame (via the coordinate transformation $\theta \mapsto \theta-\bar{\omega} t$ ) the non-uniform Kuramoto model (8) can be written as the consensus time-varying consensus protocol

$$
\dot{\theta}_{i}(t)=-\sum_{j=1}^{n} a_{i j}(t)\left(\theta_{i}(t)-\theta_{j}(t)\right),
$$

Statement 1) follows directly along the lines of the proof of statement 1) in Theorem V.1. In the case of symmetric coupling $P=P^{T}$, the phase dynamics (43) can be reformulated as a symmetric time-varying consensus protocol with strictly positive weights $w_{i j}(t)=P_{i j} \operatorname{sinc}\left(\theta_{i}(t)-\theta_{j}(t)\right)$ and multiple rates $D_{i}$ as

$$
\frac{d}{d t} D \theta=-L\left(w_{i j}(t)\right) \theta
$$

Statement 2) now follows directly along the lines of the proof of statement 2) in Theorem V.1. 4

The main result Theorem III.2 can be proved now as a corollary of Theorem V.3 and Theorem IV.2.

Proof of Theorem III.2: The assumptions of Theorem III.2 correspond exactly to the assumptions of Theorem V.3 and statements 1) and 2) of Theorem III.2 follow trivially from Theorem V.3.

Since the non-uniform Kuramoto model synchronizes exponentially and achieves phase cohesiveness in $\bar{\Delta}\left(\gamma_{\min }\right) \subsetneq \Delta\left(\pi / 2-\varphi_{\max }\right)$, it follows from Lemma IV.1 that the grounded non-uniform Kuramoto dynamics (11) converge exponentially to a stable fixed point $\delta_{\infty}$. Moreover, $\delta(0)=\operatorname{grnd}(\theta(0))$ is bounded and thus necessarily in a compact subset of the region of attraction of the fixed point $\delta_{\infty}$. Thus, the assumptions of Theorem IV.2 are satisfied. Statements 3) and 4) of Theorem III.2 follow from Theorem IV.2, where we made the following changes: the approximation errors (14)-(15) are expressed as the approximation errors (10) in $\theta$-coordinates, we stated only the case $\epsilon<\epsilon^{*}$ and $t \geq t_{b}>0$, we reformulated $h(\bar{\delta}(t))=D^{-1} Q(\bar{\theta}(t))$, and weakened the dependence of $\epsilon$ on $\Omega_{\delta}$ to a dependence on $\theta(0)$.

\footnotetext{
${ }^{3}$ This weighted average of angles is geometrically well defined for $\theta(0) \in \Delta(\pi)$.

${ }^{4}$ The proof of Theorem V.5 can be extended for $H D^{-1} \omega=\mathbf{0}$ and $X=\mathbf{0}$ to show statement 2) of Theorem V.10 with a slightly different worst-case synchronization frequency than (42).
} 


\section{Vi. Simulation Results}

Figure 3 shows a simulation of the power network model (3) with $n=10$ generators and the corresponding non-uniform Kuramoto model (8), where all initial angles $\theta(0)$ are clustered with exception of the first one (red curves) and the initial frequencies are chosen as $\dot{\theta}(0) \in$ uni $(-0.1,0.1) \mathrm{rad} / \mathrm{s}$, i.e., randomly from a uniform distribution over $[-0.1,0.1]$. Additionally, at two-third of the simulation interval a transient high frequency disturbance is introduced at $\omega_{n-1}$ (yellow curve). For illustration, relative angular coordinates are defined as $\delta_{i}(t)=\theta_{i}(t)-\theta_{n}(t), i \in\{1, \ldots, n-1\}$. The system parameters satisfy $\omega_{i} \in \operatorname{uni}(0,10), P_{i j} \in \operatorname{uni}(0.7,1.2)$, and $\tan \left(\varphi_{i j}\right) \in \operatorname{uni}(0,0.25)$ matching data found in [11], [40], [41].

For the simulation in Figure 3(a), we chose $M_{i} \in \operatorname{uni}(2,12) s /\left(2 \pi f_{0}\right)$ and $D_{i} \in \operatorname{uni}(20,30) s /\left(2 \pi f_{0}\right)$ resulting in the rather large perturbation parameter $\epsilon=0.58 \mathrm{~s}$. The synchronization conditions of Theorem III. 2 are satisfied, and the angles $\bar{\delta}(t)$ of the non-uniform Kuramoto model synchronize very fast from the non-synchronized initial conditions (within $0.05 \mathrm{~s}$ ), and the disturbance around $t=2 \mathrm{~s}$ does not severely affect the synchronization dynamics. The same findings hold for the quasi-steady state $h(\bar{\delta})$ depicting the frequencies of the non-uniform Kuramoto model, where the the disturbance at angle $n-1$ (yellow curve) acts directly without being integrated. Since $\epsilon$ is large the power network trajectories $(\delta(t), \dot{\theta}(t))$ show the expected underdamped behavior and synchronize with second-order dynamics. As expected, the disturbance at $t=2 \mathrm{~s}$ does not affect the second order power network $\delta$-dynamics as much as the firstorder non-uniform Kuramoto $\bar{\delta}$-dynamics. Nevertheless, after the initial and mid-simulation transients the singular perturbation errors $\delta(t)-\bar{\delta}(t)$ and $\theta(t)-h(\bar{\delta}(t))$ quickly become small and ultimately converge.

Figure 3(b) shows the exact same simulation as in Figure 3(a), except that the simulation time is halved, the inertia are $M_{i} \in \operatorname{uni}(2,6) s /\left(2 \pi f_{0}\right)$, and the damping is chosen uniformly as $D_{i}=30 s /\left(2 \pi f_{0}\right)$, which gives the small perturbation parameter $\epsilon=0.18 \mathrm{~s}$. The resulting power network dynamics $(\delta(t), \dot{\theta}(t))$ are strongly damped (note the different time scales), and the non-uniform Kuramoto dynamics $\bar{\delta}(t)$ and the quasi-steady state $h(\bar{\delta}(t))$ have slower time constants. As expected, the singular perturbation errors remain smaller during transients and converge faster than in the weakly damped case in Figure 3(a).

\section{CONCLUSIONS}

This paper studied the synchronization and transient stability problem for a power network. A novel approach leads to purely algebraic conditions, under which a network-reduced power system model is transiently stable depending on network parameters and initial phase differences. Our technical approach is based on the assumption that each generator is highly overdamped due to local excitation control. The resulting singular perturbation analysis leads to the successful marriage of transient stability in power networks, Kuramoto oscillators, and consensus protocols. As a result, the transient stability analysis of a power network model reduces to the synchronization analysis of non-uniform Kuramoto oscillators. The study of generalized coupled oscillator models is an interesting mathematical problem in its own right and was tackled by combining and extending different techniques from all three mentioned areas. 

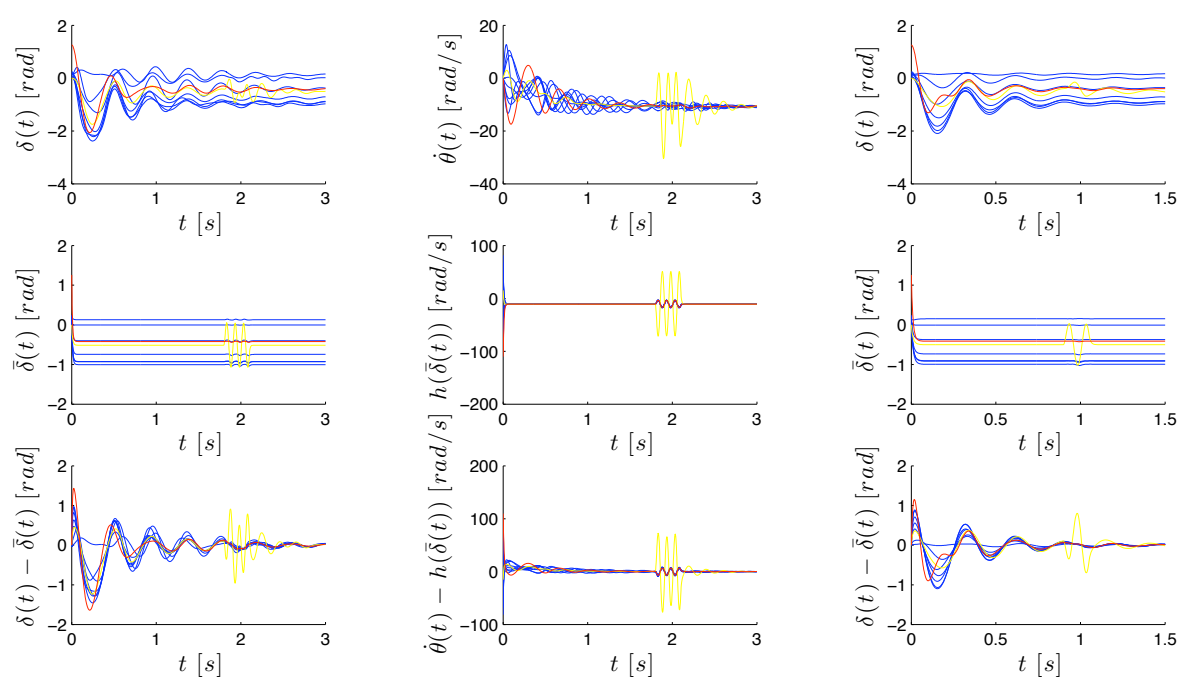

(a) Weakly damped simulation with $\epsilon=0.58 \mathrm{~s}$
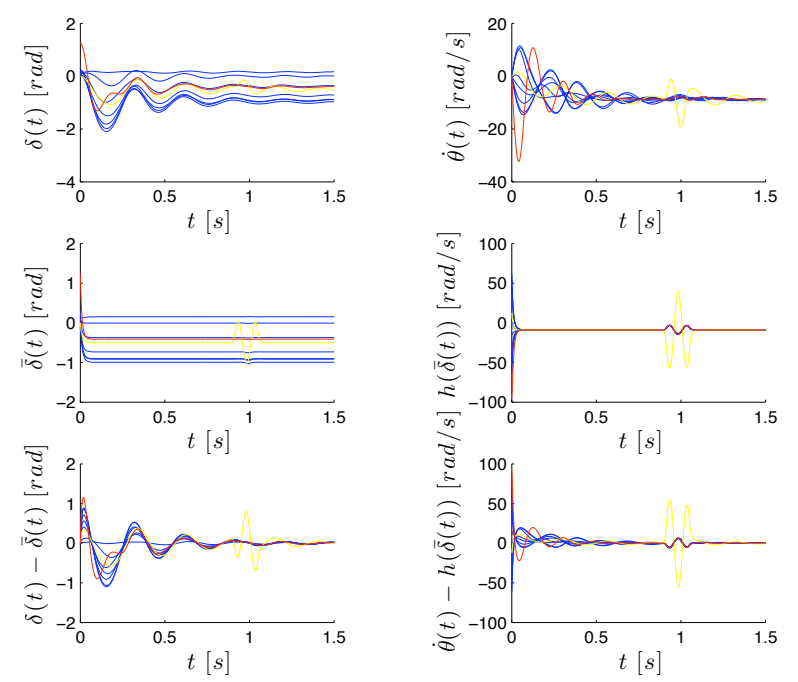

(b) Strongly damped simulation with $\epsilon=0.18 \mathrm{~s}$

Fig. 3. Simulation of the power network model (3) and the non-uniform Kuramoto model (8)

The presented approach to synchronization in power networks offers easily checkable conditions and an entirely new perspective on the transient stability problem. The authors are aware that the derived conditions are not yet competitive with the sophisticated numerical algorithms developed by the power systems community. To render our results applicable to real power systems, tighter synchronization conditions have to be developed, the region of attraction has to be characterized more accurately, and more realistic power network models have to be considered. The authors' ongoing work addresses the last point and extends the presented analysis to structure-preserving power network models.

Finally, the revealed relationship between power networks, Kuramoto oscillators, and consensus algorithms gives rise to various exciting research directions at the interface between these areas.

\section{REFERENCES}

[1] F. Dörfler and F. Bullo, "Synchronization and transient stability in power networks and non-uniform Kuramoto oscillators," in American Control Conference, Baltimore, MD, USA, June 2010, pp. 930-937.

[2] P. Pourbeik, P. S. Kundur, and C. M. Taylor, "The anatomy of a power grid blackout - root causes and dynamics of recent major blackouts," IEEE Power and Energy Magazine, vol. 4, no. 5, pp. 22-29, 2006.

[3] V. Vittal, "Transient stability and control of large scale power systems," Power Systems Engineering Research (PSERC) Background Paper, Sept. 2003.

[4] H.-D. Chiang, F. F. Wu, and P. P. Varaiya, "Foundations of the potential energy boundary surface method for power system transient stability analysis," IEEE Transactions on Circuits and Systems, vol. 35, no. 6, pp. 712-728, 1988.

[5] T. Athay, R. Podmore, and S. Virmani, "A practical method for the direct analysis of transient stability," IEEE Transactions on Power Apparatus and Systems, vol. 98, no. 2, pp. 573-584, 1979.

[6] J. Baillieul and C. Byrnes, "Geometric critical point analysis of lossless power system models," IEEE Transactions on Circuits and Systems, vol. 29, no. 11, pp. 724-737, 1982. 
[7] H.-D. Chiang and C. C. Chu, "Theoretical foundation of the BCU method for direct stability analysis of network-reduction power system models with small transfer conductances," IEEE Transactions on Circuits and Systems I: Fundamental Theory and Applications, vol. 42, no. 5, pp. 252-265, 1995.

[8] N. G. Bretas and L. F. C. Alberto, "Lyapunov function for power systems with transfer conductances: extension of the invariance principle," IEEE Transactions on Power Systems, vol. 18, no. 2, pp. 769-777, 2003.

[9] F. H. J. R. Silva, L. F. C. Alberto, J. B. A. London Jr, and N. G. Bretas, "Smooth perturbation on a classical energy function for lossy power system stability analysis," IEEE Transactions on Circuits and Systems I: Fundamental Theory and Applications, vol. 52, no. 1, pp. 222-229, 2005.

[10] P. Varaiya, F. F. Wu, and R. L. Chen, "Direct methods for transient stability analysis of power systems: Recent results," Proceedings of the IEEE, vol. 73, no. 12, pp. 1703-1715, 1985.

[11] M. A. Pai, Energy Function Analysis for Power System Stability. Kluwer Academic Publishers, 1989.

[12] H.-D. Chiang, C. C. Chu, and G. Cauley, "Direct stability analysis of electric power systems using energy functions: theory, applications, and perspective," Proceedings of the IEEE, vol. 83, no. 11, pp. 1497-1529, 1995.

[13] L. F. C. Alberto, F. H. J. R. Silva, and N. G. Bretas, "Direct methods for transient stability analysis in power systems: state of art and future perspectives," in IEEE Power Tech Proceedings, Porto, Portugal, Sept. 2001.

[14] D. J. Hill and G. Chen, "Power systems as dynamic networks," in IEEE Int. Symposium on Circuits and Systems, Kos, Greece, May 2006, pp. 722-725.

[15] R. Olfati-Saber, J. A. Fax, and R. M. Murray, "Consensus and cooperation in networked multi-agent systems," Proceedings of the IEEE, vol. 95, no. 1, pp. 215-233, 2007.

[16] W. Ren, R. W. Beard, and E. M. Atkins, "Information consensus in multivehicle cooperative control: Collective group behavior through local interaction," IEEE Control Systems Magazine, vol. 27, no. 2, pp. 71-82, 2007.

[17] F. Bullo, J. Cortés, and S. Martínez, Distributed Control of Robotic Networks, ser. Applied Mathematics Series. Princeton University Press, 2009, available at http://www.coordinationbook.info.

[18] L. Moreau, "Stability of multiagent systems with time-dependent communication links," IEEE Transactions on Automatic Control, vol. 50, no. 2, pp. 169-182, 2005.

[19] Z. Lin, B. Francis, and M. Maggiore, "State agreement for continuous-time coupled nonlinear systems," SIAM Journal on Control and Optimization, vol. 46, no. 1, pp. 288-307, 2007.

[20] D. J. Hill and J. Zhao, "Global synchronization of complex dynamical networks with non-identical nodes," in IEEE Conf. on Decision and Control, Cancún, México, Dec. 2008, pp. 817-822.

[21] M. Arcak, "Passivity as a design tool for group coordination," IEEE Transactions on Automatic Control, vol. 52, no. 8, pp. 1380-1390, 2007.

[22] A. Arenas, A. Díaz-Guilera, J. Kurths, Y. Moreno, and C. Zhou, "Synchronization in complex networks," Physics Reports, vol. 469, no. 3, pp. 93-153, 2008.

[23] Y. Kuramoto, Chemical Oscillations, Waves, and Turbulence. Dover Publications, 2003.

[24] F. De Smet and D. Aeyels, "Partial entrainment in the finite Kuramoto-Sakaguchi model," Physica D: Nonlinear Phenomena, vol. 234, no. 2, pp. 81-89, 2007.

[25] J. L. van Hemmen and W. F. Wreszinski, "Lyapunov function for the Kuramoto model of nonlinearly coupled oscillators," Journal of Statistical Physics, vol. 72, no. 1, pp. 145-166, 1993.

[26] K. Wiesenfeld, P. Colet, and S. H. Strogatz, "Frequency locking in Josephson arrays: Connection with the Kuramoto model," Physical Review E, vol. 57, no. 2, pp. 1563-1569, 1998.

[27] R. E. Mirollo and S. H. Strogatz, "The spectrum of the locked state for the Kuramoto model of coupled oscillators," Physica D: Nonlinear Phenomena, vol. 205, no. 1-4, pp. 249-266, 2005.

[28] E. Canale and P. Monzón, "Almost global synchronization of symmetric Kuramoto coupled oscillators," in Systems Structure and Control. InTech Education and Publishing, 2008, ch. 8, pp. 167-190.

[29] D. Aeyels and J. A. Rogge, "Existence of partial entrainment and stability of phase locking behavior of coupled oscillators," Progress on Theoretical Physics, vol. 112, no. 6, pp. 921-942, 2004.

[30] S. H. Strogatz, "From Kuramoto to Crawford: Exploring the onset of synchronization in populations of coupled oscillators," Physica D: Nonlinear Phenomena, vol. 143, no. 1, pp. 1-20, 2000. 
[31] J. A. Acebrón, L. L. Bonilla, C. J. P. Vicente, F. Ritort, and R. Spigler, "The Kuramoto model: A simple paradigm for synchronization phenomena," Reviews of Modern Physics, vol. 77, no. 1, pp. 137-185, 2005.

[32] N. Chopra and M. W. Spong, "On exponential synchronization of Kuramoto oscillators," IEEE Transactions on Automatic Control, vol. 54, no. 2, pp. 353-357, 2009.

[33] A. Jadbabaie, N. Motee, and M. Barahona, "On the stability of the Kuramoto model of coupled nonlinear oscillators," in American Control Conference, Boston, MA, June 2004, pp. 4296-4301.

[34] G. Filatrella, A. H. Nielsen, and N. F. Pedersen, "Analysis of a power grid using a Kuramoto-like model," The European Physical Journal B, vol. 61, no. 4, pp. 485-491, 2008.

[35] V. Fioriti, S. Ruzzante, E. Castorini, E. Marchei, and V. Rosato, "Stability of a distributed generation network using the Kuramoto models," in Critical Information Infrastructure Security, ser. Lecture Notes in Computer Science. Springer, 2009, pp. 14-23.

[36] D. Subbarao, R. Uma, B. Saha, and M. V. R. Phanendra, "Self-organization on a power system," IEEE Power Engineering Review, vol. 21, no. 12, pp. 59-61, 2001.

[37] H. A. Tanaka, A. J. Lichtenberg, and S. Oishi, "Self-synchronization of coupled oscillators with hysteretic responses," Physica D: Nonlinear Phenomena, vol. 100, no. 3-4, pp. 279-300, 1997.

[38] H.-D. Chiang, Direct Methods for Stability Analysis of Electric Power Systems. Wiley, 2010.

[39] G. S. Schmidt, U. Münz, and F. Allgöwer, "Multi-agent speed consensus via delayed position feedback with application to Kuramoto oscillators," in European Control Conference, Budapest, Hungary, Aug. 2009, pp. 2464-2469.

[40] P. M. Anderson and A. A. Fouad, Power System Control and Stability. Iowa State University Press, 1977.

[41] P. Kundur, Power System Stability and Control. McGraw-Hill, 1994.

[42] F. Dörfler and F. Bullo, "Kron reduction of graphs with applications to electrical networks," SIAM Review, Feb. 2011, submitted.

[43] R. Ortega, M. Galaz, A. Astolfi, Y. Sun, and T. Shen, "Transient stabilization of multimachine power systems with nontrivial transfer conductances," IEEE Transactions on Automatic Control, vol. 50, no. 1, pp. 60-75, 2005.

[44] P. W. Sauer and M. A. Pai, Power System Dynamics and Stability. Prentice Hall, 1998.

[45] L. F. C. Alberto and N. G. Bretas, "Synchronism versus stability in power systems," Electrical Power and Energy Systems, vol. 21, no. 4, pp. 261-267, 1999.

[46] H.-D. Chiang, "Study of the existence of energy functions for power systems with losses," IEEE Transactions on Circuits and Systems, vol. 36, no. 11, pp. 1423-1429, 1989.

[47] R. De Luca, "Strongly coupled overdamped pendulums," Revista Brasileira de Ensino de Física, vol. 30, pp. 4304-4304, 2008.

[48] S. Ha, C. Lattanzio, B. Rubino, and M. Slemrod, "Flocking and synchronization of particle models," Quarterly Applied Mathematics, vol. 69, pp. 91-103, 2011.

[49] L. F. C. Alberto and N. G. Bretas, "Required damping to assure multiswing transient stability: the SMIB case," International Journal of Electrical Power \& Energy Systems, vol. 22, no. 3, pp. 179-185, 2000.

[50] C. Chu and H. Chiang, "Boundary properties of the BCU method for power system transient stability assessment," in IEEE Int. Symposium on Circuits and Systems, Paris, France, May 2010, pp. 3453-3456.

[51] H. K. Khalil, Nonlinear Systems, 3rd ed. Prentice Hall, 2002.

[52] J. Baillieul, "The critical point analysis of electric power systems," in IEEE Conf. on Decision and Control, Dec. 1984, pp. 154-159.

[53] L. Moreau, "Stability of continuous-time distributed consensus algorithms," Sept. 2004, available at http://arxiv.org/abs/math/0409010.

[54] C. D. Meyer, Matrix Analysis and Applied Linear Algebra. SIAM, 2001.

[55] F. Dörfler and F. Bullo, "On the critical coupling for Kuramoto oscillators," SIAM Journal on Applied Dynamical Systems, Nov. 2010, submitted. 


\section{AppendiX: Alternative Synchronization Conditions}

In this appendix we briefly comment on alternative bounding methods in the proof of Theorem V.3 and how they affect the triplet of the synchronization condition (9), the estimate for the region of attraction $\Delta\left(\gamma_{\max }\right)$, and the ultimate phase cohesive set $\bar{\Delta}\left(\gamma_{\min }\right)$. We will state only the essential parts of the theorem statements and the corresponding proofs.

\section{A. Pairwise Bounding}

The proof of Theorem V.3 can be continued from equation (29) by bounding the right-hand side of equation (29) for each single pair $\{m, \ell\}$ rather than for all $m, \ell \in\{1, \ldots, n\}$. Such a pairwise bounding results in $n(n-1) / 2$ pairwise synchronization conditions and the worst multiplicative gap over all conditions determines the estimates for the region of attraction $\Delta\left(\gamma_{\max }\right)$ and the ultimate phase cohesive set $\bar{\Delta}\left(\gamma_{\min }\right)$. In short, tighter bounds are traded off for complexity. The resulting theorem statement is as follows.

Theorem VIII.1 (Synchronization condition I) Consider the non-uniform Kuramoto-model (8), where the graph induced by $P=P^{T}$ is complete. Assume that the minimal lossless coupling of any oscillator pair $\{m, \ell\}$ to the network is larger than a critical value, i.e., for every $m, \ell \in\{1, \ldots, n\}, m \neq \ell$,

$$
\begin{aligned}
\Gamma_{m \ell}:=\sum_{k=1}^{n} \min _{i \in\{m, \ell\} \backslash\{k\}}\left\{\frac{P_{i k}}{D_{i}} \cos \left(\varphi_{i k}\right)\right\}> \\
\Gamma_{m \ell}^{\text {critical }}:=\frac{1}{\cos \left(\varphi_{\max }\right)} \cdot\left(\left|\frac{\omega_{m}}{D_{m}}-\frac{\omega_{\ell}}{D_{\ell}}\right|+\sum_{k=1}^{n}\left(\frac{P_{m k}}{D_{m}} \sin \left(\varphi_{m k}\right)+\frac{P_{\ell k}}{D_{\ell}} \sin \left(\varphi_{\ell k}\right)\right)\right) .
\end{aligned}
$$

Accordingly, define $\gamma_{\min } \in\left[0, \pi / 2-\varphi_{\max }\left[\right.\right.$ and $\left.\left.\gamma_{\max } \in\right] \pi / 2, \pi\right]$ as unique solutions to the equations $\sin \left(\gamma_{\min }\right)=\sin \left(\gamma_{\max }\right)=\cos \left(\varphi_{\max }\right) \max _{m, \ell}\left\{\Gamma_{m \ell}^{\text {critical }} / \Gamma_{m \ell}\right\}$. Then $\ldots$

Proof of Theorem VIII.1: ... [see proof of Theorem V.3] ...

In summary, $D^{+} V(\theta(t))$ in (29) can be upper bounded by the simple expression

$$
D^{+} V(\theta(t)) \leq \frac{\omega_{m}}{D_{m}}-\frac{\omega_{\ell}}{D_{\ell}}-\sum_{k=1}^{n} \min _{i \in\{m, \ell\} \backslash\{k\}}\left\{a_{i k}\right\} \sin (\gamma)+\sum_{k} b_{\ell k}+\sum_{k} b_{m k} .
$$

It follows that $V(\theta(t))$ is non-increasing for all $\theta(t) \in \bar{\Delta}(\gamma)$ and for all pairs $\{m, \ell\}$ if

$$
\Gamma_{m \ell} \sin (\gamma) \geq \cos \left(\varphi_{\max }\right) \Gamma_{m \ell}^{\text {critical }},
$$

where $\Gamma_{m \ell}$ and $\Gamma_{m \ell}^{\text {critical }}$ are defined in (45). The left-hand side of (46) is a strictly concave function of $\gamma \in[0, \pi]$. Thus, there exists an open set of arc lengths $\gamma$ including $\gamma^{*}=\pi / 2-\varphi_{\max }$ satisfying equation (46) if and only if equation (46) is true at $\gamma^{*}=\pi / 2-\varphi_{\max }$ with the strict inequality sign, which corresponds to condition (45) in the statement of Theorem VIII.1. Additionally, if these two equivalent statements are true, then $V(\theta(t))$ is non-increasing in $\bar{\Delta}(\gamma)$ for all $\gamma \in\left[\gamma_{\min }, \gamma_{\max }\right]$, where $\gamma_{\min } \in\left[0, \pi / 2-\varphi_{\max }\left[\right.\right.$ and $\left.\left.\gamma_{\max } \in\right] \pi / 2, \pi\right]$ satisify inequality (46) for all pairs $\{m, \ell\} \ldots$ 


\section{B. Pairwise Concavity-Based Bounding}

The bounding in the proof of Theorem V.3 can be further tightened by cocavity-based arguments. This bounding results in $n(n-1) / 2$ pairwise synchronization conditions and $n(n-1)$ equations determining the estimates for the region of attraction $\Delta\left(\gamma_{\max }\right)$ and the ultimate phase cohesive set $\bar{\Delta}\left(\gamma_{\min }\right)$. Again, tighter bounds are traded off for increasing complexity. The resulting theorem statement is as follows.

Theorem VIII.2 (Synchronization condition I) Consider the non-uniform Kuramoto-model (8), where the graph induced by $P=P^{T}$ is complete. Assume that the minimal lossless coupling of any oscillator pair $\{m, \ell\}$ to the network is larger than a critical value, i.e., for every $m, \ell \in\{1, \ldots, n\}, m \neq \ell$,

$$
\sum_{k=1}^{n} \min _{i \in\{m, \ell\} \backslash\{k\}}\left\{\frac{P_{i k}}{D_{i}} \cos \left(\varphi_{i k}+\varphi_{\max }\right)\right\}>\Gamma_{m \ell}^{\text {critical }}:=\left|\frac{\omega_{m}}{D_{m}}-\frac{\omega_{\ell}}{D_{\ell}}\right|+\max _{i \in\{m, \ell\}}\left\{\sum_{k=1}^{n} \frac{P_{i k}}{D_{i}} \sin \left(\varphi_{i k}\right)\right\} .
$$

Accordingly, for every pair $\{m, l\}$ define $\gamma_{\min }^{m \ell} \in\left[0, \pi / 2-\varphi_{\max }\left[\right.\right.$ and $\left.\left.\gamma_{\max }^{m \ell} \in\right] \pi / 2, \pi\right]$ as unique solutions to

$$
\sum_{k=1}^{n} \min _{i \in\{m, \ell\} \backslash\{k\}}\left\{\frac{P_{i k}}{D_{i}} \sin \left(\gamma_{\min }^{m \ell}-\varphi_{i k}\right)\right\}=\sum_{k=1}^{n} \min _{i \in\{m, \ell\} \backslash\{k\}}\left\{\frac{P_{i k}}{D_{i}} \sin \left(\gamma_{\max }^{m \ell}+\varphi_{i k}\right)\right\}=\Gamma_{m \ell}^{\text {critical }},
$$

and let $\gamma_{\min }:=\max _{m, \ell}\left\{\gamma_{\min }^{m \ell}\right\}$ and $\gamma_{\max }:=\min _{m, \ell}\left\{\gamma_{\max }^{m \ell}\right\}$. Then ...

Proof of Theorem VIII.2: ... [see proof of Theorem V.3] ...

Written out in components $D^{+} V(\theta(t))$ (in the non-expanded form (8)) takes the form

$$
D^{+} V(\theta(t))=\frac{\omega_{m}}{D_{m}}-\frac{\omega_{\ell}}{D_{\ell}}-\sum_{k=1}^{n} \frac{P_{m k}}{D_{m}} \sin \left(\theta_{m}(t)-\theta_{k}(t)+\varphi_{m k}\right)+\frac{P_{\ell k}}{D_{\ell}} \sin \left(\theta_{k}(t)-\theta_{\ell}(t)-\varphi_{\ell k}\right) .
$$

In the following we abbreviate the summand on the right-hand side of (49) as $f_{k}\left(\theta_{k}(t)\right):=\frac{P_{m k}}{D_{m}} \sin \left(\theta_{m}(t)-\right.$ $\left.\theta_{k}(t)+\varphi_{m k}\right)+\frac{P_{\ell k}}{D_{\ell}} \sin \left(\theta_{k}(t)-\theta_{\ell}(t)-\varphi_{\ell k}\right)$ and aim at a least conservative bounding of $f_{k}\left(\theta_{k}(t)\right)$. There are different ways to continue from here - the following approach is based on concavity.

Since $f_{k}\left(\theta_{k}\right)$ is the sum of two shifted concave sine functions of $\theta_{k} \in\left[\theta_{\ell}, \theta_{m}\right]$ (with same period and shifted by strictly less than $\pi / 2), f_{k}\left(\theta_{k}\right)$ is again concave sine function of $\theta_{k} \in\left[\theta_{\ell}, \theta_{m}\right]$ and necessarily achieves its minimum at the boundary $\theta_{k} \in\left\{\theta_{\ell}, \theta_{m}\right\}$. If $\operatorname{argmin}_{\theta_{k} \in\left[\theta_{m}, \theta_{\ell}\right]} f_{k}\left(\theta_{k}\right)=\theta_{\ell}$, then

$$
f_{k}\left(\theta_{k}(t)\right) \geq f_{k}^{1}(\gamma):=\frac{P_{m k}}{D_{m}} \sin \left(\gamma+\varphi_{m k}\right)-\frac{P_{\ell k}}{D_{\ell}} \sin \left(\varphi_{\ell k}\right)
$$

and otherwise for $\operatorname{argmin}_{\theta_{k} \in\left[\theta_{m}, \theta_{\ell}\right]} f_{k}\left(\theta_{k}\right)=\theta_{m}$

$$
f_{k}\left(\theta_{k}(t)\right) \geq f_{k}^{2}(\gamma):=\frac{P_{\ell k}}{D_{\ell}} \sin \left(\gamma-\varphi_{\ell k}\right)+\frac{P_{m k}}{D_{m}} \sin \left(\varphi_{m k}\right),
$$

where $f_{k}^{1}$ and $f_{k}^{2}$ are functions from $[0, \pi]$ to $\mathbb{R}$. In the following let $f_{k}^{3}:[0, \pi] \rightarrow \mathbb{R}$ be defined by

$$
f_{k}^{3}(\gamma):=\min \left\{\frac{P_{m k}}{D_{m}} \sin \left(\gamma+\varphi_{m k}\right), \frac{P_{\ell k}}{D_{\ell}} \sin \left(\gamma-\varphi_{\ell k}\right)\right\}-\frac{P_{\ell k}}{D_{\ell}} \sin \left(\varphi_{\ell k}\right) .
$$

Since $f_{k}(\theta(t)) \geq f_{k}^{3}(\gamma)$ for all $\theta_{k}(t) \in\left[\theta_{m}(t), \theta_{\ell}(t)\right]$, the derivative (49) is upper-bounded by

$$
D^{+} V(\theta(t))=\frac{\omega_{m}}{D_{m}}-\frac{\omega_{\ell}}{D_{\ell}}-\sum_{k} f_{k}^{3}(\gamma)
$$


It follows that $V(\theta(t))$ is non-increasing for all $\theta(t) \in \bar{\Delta}(\gamma)$ and for all pairs $\{m, \ell\}$ if for all $\{m, \ell\}$

$$
\sum_{k=1}^{n} \min _{i \in\{m, \ell\} \backslash\{k\}}\left\{\frac{P_{i k}}{D_{i}} \sin \left(\gamma+\varphi_{i k}\right), \frac{P_{i k}}{D_{i}} \sin \left(\gamma-\varphi_{i k}\right)\right\} \geq\left|\frac{\omega_{m}}{D_{m}}-\frac{\omega_{\ell}}{D_{\ell}}\right|+\max _{i \in\{m, \ell\}}\left\{\sum_{k=1}^{n} \frac{P_{i k}}{D_{i}} \sin \left(\varphi_{i k}\right)\right\} .
$$

Note that the minimizing summand on the left-hand side of (50) is $\min _{i \in\{m, \ell\} \backslash\{k\}}\left\{P_{i k} \sin \left(\gamma-\varphi_{i k}\right) / D_{i}\right\}$ for $\gamma<\pi / 2, \min _{i \in\{m, \ell\} \backslash\{k\}}\left\{P_{i k} \sin \left(\gamma+\varphi_{i k}\right) / D_{i}\right\}$ for $\gamma>\pi / 2$, and it achieves its maximum value $\min _{i \in\{m, \ell\} \backslash\{k\}}\left\{P_{i k} \cos \left(\varphi_{i k}\right) / D_{i}\right\}$ for $\gamma=\pi / 2$. In particular, there exists an open set of arc lengths $\gamma$ for including $\gamma^{*}=\pi / 2-\varphi_{\max }$ for which $V(\theta(t))$ is non-increasing in $\bar{\Delta}(\gamma)$ if and only if inequality (50) is strictly satisfied for $\gamma^{*}=\pi / 2-\varphi_{\max }$. In this case, define $\gamma_{\min }^{m \ell} \in\left[0, \pi / 2-\varphi_{\max }\left[\right.\right.$ and $\left.\left.\gamma_{\max }^{m \ell} \in\right] \pi / 2, \pi\right]$ as the two unique solutions to equation (50) with equality sign, which is equivalent to equation (48). Then $V(\theta(t))$ is non-increasing in $\bar{\Delta}(\gamma)$ for all $\gamma \in\left[\gamma_{\min }^{m \ell}, \gamma_{\max }^{m \ell}\right]$. Finally define $\gamma_{\min }$ and $\gamma_{\max }$ as the maximum and minimum values of $\gamma_{\min }^{m \ell}$ and $\gamma_{\max }^{m \ell}$ over all pairs $\{m, \ell\} \ldots$

\section{Adding and Subtracting the Lossless Coupling}

The last possible bounding we explore is restricted to the set of initial conditions in $\Delta\left(\pi / 2-\varphi_{\max }\right)$ and results in a simple, scalar, and intuitive but very conservative synchronization condition. Instead of bounding the right-hand side of $D^{+} V(\theta(t))$ directly, we add and subtract the lossless coupling in the proof of Theorem V.3. The resulting theorem statement is as follows.

Theorem VIII.3 (Synchronization condition I) Consider the non-uniform Kuramoto-model (8), where the graph induced by $P=P^{T}$ is complete. Assume that the minimal coupling is larger than a critical value, i.e., for every $i, j \in\{1, \ldots, n\}$

$$
P_{\min }>P_{\text {critical }}:=\frac{D_{\max }}{n \cos \left(\varphi_{\max }\right)}\left(\max _{\{i, j\}}\left|\frac{\omega_{i}}{D_{i}}-\frac{\omega_{j}}{D_{j}}\right|+\max _{i} \sum_{j=1}^{n} \frac{P_{i j}}{D_{i}} \sin \left(\varphi_{i j}\right)\right) .
$$

Accordingly, define $\gamma_{\min }=\arcsin \left(\cos \left(\varphi_{\max }\right) P_{\text {critical }} / P_{\min }\right)$ taking value in $\left[0, \pi / 2-\varphi_{\max }\left[\right.\right.$ and $\gamma_{\max }=$ $\pi / 2-\varphi_{\max }$. Then ...

Proof of Theorem VIII.3: ... see proof of Theorem V.3 ...

Written out in components (in the non-expanded form (8)) $D^{+} V(\theta(t))$ takes the form

$$
D^{+} V(\theta(t))=\frac{\omega_{m}}{D_{m}}-\frac{\omega_{\ell}}{D_{\ell}}-\sum_{k=1}^{n}\left(\frac{P_{m k}}{D_{m}} \sin \left(\theta_{m}(t)-\theta_{k}(t)+\varphi_{m k}\right)-\frac{P_{\ell k}}{D_{\ell}} \sin \left(\theta_{\ell}(t)-\theta_{k}(t)+\varphi_{\ell k}\right)\right) \text {. }
$$

Adding and subtracting the coupling with zero phase shifts yields

$$
\begin{aligned}
D^{+} V(\theta(t))= & \frac{\omega_{m}}{D_{m}}-\frac{\omega_{\ell}}{D_{\ell}}-\sum_{k=1}^{n}\left(\frac{P_{m k}}{D_{m}} \sin \left(\theta_{m}(t)-\theta_{k}(t)\right)+\frac{P_{\ell k}}{D_{\ell}} \sin \left(\theta_{k}(t)-\theta_{\ell}(t)\right)\right) \\
& -\sum_{k=1}^{n} \frac{P_{m k}}{D_{m}}\left(\sin \left(\theta_{m}(t)-\theta_{k}(t)+\varphi_{m k}\right)-\sin \left(\theta_{m}(t)-\theta_{k}(t)\right)\right) \\
& \left.+\sum_{k=1}^{n} \frac{P_{\ell k}}{D_{\ell}}\left(\sin \left(\theta_{\ell}(t)-\theta_{k}(t)\right)+\varphi_{\ell k}\right)+\sin \left(\theta_{k}(t)-\theta_{\ell}(t)\right)\right)
\end{aligned}
$$


Since both sinusoidal terms in the first sum are strictly positive, they can be lower-bounded as

$$
\frac{P_{m k}}{D_{m}} \sin \left(\theta_{m}(t)-\theta_{k}(t)\right)+\frac{P_{\ell k}}{D_{\ell}} \sin \left(\theta_{k}(t)-\theta_{\ell}(t)\right) \geq \frac{P_{\min }}{D_{\max }}\left(\sin \left(\theta_{m}(t)-\theta_{k}(t)\right)+\sin \left(\theta_{k}(t)-\theta_{\ell}(t)\right)\right) .
$$

In the following we apply classic trigonometric arguments from the Kuramoto literature [32], [24], [39]. The identity $\sin (x)+\sin (y)=2 \sin \left(\frac{x+y}{2}\right) \cos \left(\frac{x-y}{2}\right)$ leads to the further simplifications

$$
\begin{aligned}
\sin \left(\theta_{m}(t)-\theta_{k}(t)\right)+\sin \left(\theta_{k}(t)-\theta_{\ell}(t)\right) & =2 \sin \left(\frac{\theta_{m}(t)-\theta_{\ell}(t)}{2}\right) \cos \left(\frac{\theta_{m}(t)+\theta_{\ell}(t)}{2}-\theta_{k}(t)\right), \\
\sin \left(\theta_{m}(t)-\theta_{k}(t)+\varphi_{m k}\right)+\sin \left(\theta_{k}(t)-\theta_{m}(t)\right) & =2 \sin \left(\frac{\varphi_{m k}}{2}\right) \cos \left(\theta_{m}(t)-\theta_{k}(t)+\frac{\varphi_{m k}}{2}\right), \\
\sin \left(\theta_{\ell}(t)-\theta_{k}(t)+\varphi_{\ell k}\right)+\sin \left(\theta_{k}(t)-\theta_{\ell}(t)\right) & =2 \sin \left(\frac{\varphi_{\ell k}}{2}\right) \cos \left(\theta_{\ell}(t)-\theta_{k}(t)+\frac{\varphi_{\ell k}}{2}\right) .
\end{aligned}
$$

Note that the right-hand side of (52) is a convex function of $\theta_{k} \in\left[\theta_{\ell}, \theta_{m}\right]$ (see Proof of Theorem VIII.2) and accordingly achieves its maximum at the boundary for $\theta_{k} \in\left\{\theta_{\ell}, \theta_{m}\right\}$ Therefore, $D^{+} V(\theta(t))$ is upper bounded by

$$
\begin{aligned}
D^{+} V(\theta(t)) \leq \max _{\{i, j\}}\left|\frac{\omega_{i}}{D_{i}}-\frac{\omega_{j}}{D_{j}}\right|-\frac{P_{\min }}{D_{\max }} \sum_{k=1}^{n} 2 \sin \left(\frac{\gamma}{2}\right) \cos \left(\frac{\gamma}{2}\right) \\
\quad-\sum_{k=1}^{n} \frac{P_{m k}}{D_{m}} 2 \sin \left(\frac{\varphi_{m k}}{2}\right) \cos \left(\gamma+\frac{\varphi_{m k}}{2}\right)+\sum_{k=1}^{n} \frac{P_{\ell k}}{D_{\ell}} 2 \sin \left(\frac{\varphi_{\ell k}}{2}\right) \cos \left(\frac{\varphi_{\ell k}}{2}\right) .
\end{aligned}
$$

Note that the second sum is strictly negative for $\gamma \in\left[0, \pi / 2-\varphi_{\max }[\right.$ and can be neglected. Moreover, in the third sum the maximum over all nodes $\ell$ can be taken. Reversing the trigonometric identity from above as $2 \sin (x) \cos (y)=\sin (x-y)+\sin (x+y)$ yields then the simple expression

$$
D^{+} V(\theta(t)) \leq \max _{\{i, j\}}\left|\frac{\omega_{i}}{D_{i}}-\frac{\omega_{j}}{D_{j}}\right|-\frac{P_{\min }}{D_{\max }} \sum_{k=1}^{n} \sin (\gamma)+\max _{i} \sum_{k=1}^{n} \frac{P_{i k}}{D_{i}} \sin \left(\varphi_{i k}\right) .
$$

It follows that the length of the arc formed by the angles is non-increasing in $\Delta(\gamma)$ if

$$
P_{\min } \sin (\gamma) \geq P_{\text {critical }} \cos \left(\varphi_{\max }\right),
$$

where $P_{\text {critical }}$ is as stated in equation (51). The left-hand side of (53) is a strictly increasing function of $\gamma \in$ $\left[0, \pi / 2-\varphi_{\max }\left[\right.\right.$. Therefore, there exists some $\gamma^{*} \in\left[0, \pi / 2-\varphi_{\max }[\right.$ satisfying equation (53) if and only if equation (53) at $\gamma=\pi / 2-\varphi_{\max }$ is true with the strict inequality sign, which corresponds to equation (51). Additionally, if these two equivalent statements are true, then there exists a unique $\gamma_{\min } \in\left[0, \pi / 2-\varphi_{\max }[\right.$ that satisfies equation (53) with the equality sign, namely $\gamma_{\min }=\arcsin \left(\cos \left(\varphi_{\max }\right) P_{\text {critical }} / P_{\min }\right) \ldots$ 